\title{
Positioning And Power Optimization For UAV- Assisted Networks In The Presence of Eavesdroppers: A Multi-Armed Bandit Approach
}

\section{Xavier Alejandro Flores Cabezas ( $\boldsymbol{\nabla}$ xavier.florescabezas@oulu.fi )}

University of Oulu https://orcid.org/0000-0001-7787-5583

Diana Pamela Moya Osorio

University of Oulu

Matti Latva-aho

University of Oulu

\section{Research}

Keywords: friendly jamming, multi-armed bandit, physical layer security, secrecy outage probability, reinforcement learning, UAV-assisted communications

Posted Date: November 1st, 2021

DOI: https://doi.org/10.21203/rs.3.rs-1013858/v1

License: (9) This work is licensed under a Creative Commons Attribution 4.0 International License. Read Full License 
Correspondence:

xavier.florescabezas@oulu.fi Centre for Wireless

Communications, University of Oulu, Oulu, Finland

Full list of author information is available at the end of the article

\begin{abstract}
Unmanned Aerial Vehicles (UAVs) are becoming increasingly attractive for the ambitious expectations for $5 \mathrm{G}$ and beyond networks due to their several benefits. Indeed, UAV-assisted communications introduce a new range of challenges and opportunities regarding the security of these networks. Thus, in this paper we explore the opportunities that UAVs can provide for physical layer security solutions. Particularly, we analize the secrecy performance of a ground wireless communication network assisted by two friendly UAV jammers in the presence of an eavesdropper. To tackle the secrecy performance of this system, we introduce a new area-based metric, the weighted secrecy coverage, that measures the improvement on the secrecy performance of a system over a certain physical area given by the introduction of friendly jamming. Herein, the optimal 3D positioning of the UAVs and the power allocation is addressed in order to maximize the WSC. For that purpose, we provide a Reinforcement Learning-based solution by modeling the positioning problem as a Multi-Armed Bandit problem over three positioning variables for the UAVs: angle, height and orbit radius. Our results show that there is a trade-off between expediency of the positioning of the UAVs to positions of better secrecy outcome and energy expenditure, and that the proposed algorithm efficiently converges into a stable state.
\end{abstract}

Keywords: friendly jamming; multi-armed bandit; physical layer security; secrecy outage probability; reinforcement learning; UAV-assisted communications

\section{Introduction}

Over the last years, the interest on the integration of unmanned aerial vehicles (UAVs) to cellular networks as new aerial nodes has exponentially increased. The advancements on cellular technologies with the fifth generation of wireless networks (5G) and the expected almost ubiquitous accessibility to these networks make UAVs to be considered as a crucial component of the sixth generation of wireless networks (6G). UAVs are expected to be deployed as aerial base stations (ABSs), access points (APs), or relays to assist terrestrial communications within the so-called UAVassisted communications. In this way, the advantageous characteristics of UAVassisted communications, such as on-demand deployment, low-cost, flexibility in network reconfiguration, and high chance of line-of-sight (LoS) links, promote the emerging of a number of novel use cases and applications in different contexts such as disaster areas, smart agriculture, traffic control, search and rescue, package delivery, among others $[1,2]$. 
Nonetheless, with all the expected technological and architectural progress for $6 \mathrm{G}$, specially with the integration of artificial intelligence (AI) into the network operation and management and the advancements of quantum computing, security becomes a highly critical aspect in order to guarantee the levels of resilience and reliability planned for 6G [3]. Physical layer security (PLS) has attracted increased attention as a mechanism to provide more robust and quantum-resistant protection to wireless networks by relying on the unique physical properties of the random and noisy wireless channels to enhance confidentiality in a flexible and adaptive manner. Thus, PLS can find a new horizon in the $6 \mathrm{G}$ era specially for the constrained scenarios of Internet of Things (IoT) applications [4, 5].

Under these circumstances, UAVs can also be exploited for the design of secure solutions in UAV-assisted communications via PLS, thus the challenges and opportunities for preventing passive and active attacks in wireless networks have been recently discussed in [6]. On one hand, UAV-assisted communications are more vulnerable to eavesdropping and jamming attacks; on the other hand, UAVs can also be used to launch more effective attacks [6]. Therefore, there is a vast research area to be exploited for providing secure wireless communications in the UAV era, and some have been already reported in the literature $[7,8,9,10,11,12,13,14,15]$.

Particularly, the introduction of UAV nodes acting as friendly jammers in order to improve the secrecy performance of wireless networks have recently risen special attention. For instance, in [7], an optimal three-dimensional (3D) deployment and jamming power of UAV-based jammer was proposed to improve the secrecy performance of a legitimate transmission between a pair of nodes for unknown eavesdropper location. In [8], the secrecy outage probability (SOP) of a UAV-based mmWave relay network in the presence of multiple eavesdroppers is investigated. Two scenarios are considered, with and without cooperative jamming, which is introduced via the destination and an external UAV. In [9], the authors studied the secrecy performance of a non-orthogonal multiple access (NOMA)-based scheme in a mmWave UAV-assisted wireless network by considering a protected-zone approach. In [10], the existence of an optimal UAV jammer location on a network with multiple eavesdroppers was proved, and the impact of the density of eavesdroppers, the transmission power of the UAV jammer, and the density of UAV jammers on the optimal location was investigated.

Moreover, the joint optimization of the transmit power and the trajectory of a UAV-based friendly jammer in a three-dimensional space was investigated in [11]. Therein, the problem of average achievable secrecy rate maximization of the secondary system was investigated for a cognitive relay network by considering the imperfect location information of ground nodes, that is the eavesdropper, secondary receiver and primary receiver. Also in [12], the secrecy rate maximization problem of a mobile user over all time slots is studied by considering a dual UAV-enabled secure communication system, where one UAV sends confidential information while the other serves as friendly jammer. Both UAVs are considered to be energy-constrained devices and the location information of eavesdroppers is imperfect. Therein, the optimization problem is solved by jointly designing the 3D trajectory of UAVs and the time allocated for recharging and jamming sending under constraints such as the maximum UAV speed, UAV collision avoidance, UAV positioning error, and UAV energy harvesting. 
More recently, machine learning (ML) approaches have been considered in order to tackle the intricacy of the optimization problems related to UAV-assisted scenarios, where there is a number of coupled variables and the complexity on the characteristics of the problems would lead to exhaustive searches or complex operations. Particularly, in [13], a deep reinforcement learning (RL) algorithm is proposed to jointly optimize the active beamforming of the UAV, the coefficients of a reflective intelligent surface (RIS) elements, and the UAV trajectory to maximize the sum secrecy rate of the legitimate users in the presence of multiple eavesdroppers of a mmWave UAV communication assisted by a RIS under imperfect channel state information (CSI). Besides, in [14], a deep learning method is employed to optimize a 3D beamformer for the transmission of confidential signal and friendly jamming in order to maximize the average secrecy rate by considering partial CSI of the legitimate UAV and eavesdropping UAV. Also, the authors in [15] considered UAVs jammers assisting a legitimate transmission between a UAV and ground nodes in presence of ground eavesdroppers. Therein, a multi-agent deep RL approach was used to maximize the secure capacity by jointly optimizing the trajectory of UAVs, the transmit power of the UAV transmitter and the jamming power of the UAV jammers. cooperative jamming approach by relying on

All in all, the employment of friendly jamming has been widely accepted as an effective manner to enable confidential transmissions in wireless networks. However, the effectiveness of friendly jamming schemes is in most cases harnessed to the perfect or partial knowledge of the CSI of the legitimate and eavesdropping links, which is hard to obtain in practice. To dive into the characterization of the effectiveness of friendly jamming in wireless networks, the authors in [16] proposed two novel areabased metrics, the jamming coverage and the jamming efficiency, in order to provide insights into the design of optimal jamming configurations by considering different levels of CSI knowledge. Later, in [17], we considered a UAV-assisted friendly jamming scheme in a wireless network in the presence of eavesdroppers. Based on the area-based metrics in [16], a novel metric, the weighted secrecy coverage (WSC), was proposed to give a better insight into the impact of friendly jamming. Thus, the optimal positioning of two UAV jammers was tackled in order to maximize the WSC. Further in [18], we proposed a zero-forcing precoding scheme for the two friendly UAV jammers in order to enhance the efficiency of the friendly jamming, thus enhancing the WSC.

Inspired by [16] and based on [17], we will advance on the state-of-the-art by studying a UAV-assisted wireless network, where two UAVs assist a legitimate ground communication between a pair of ground nodes in a confined region on a fading environment, and the 3D positioning of the UAVs is optimized in order to maximize the WSC metric. For that purpose, we model our optimization problem as a multi-armed bandit (MAB) problem and provide a RL-based solution ${ }^{[1]}$. The main contributions that carry on from the previous paper [17] are the following:

- For Rayleigh fading ground channels we find a closed form representation of a secrecy improvement metric for a generic amount of UAVs for a wireless wiretap channel with friendly UAV jammers.

${ }^{[1]}$ This is an extended version of the paper presented at the 2021 Joint EuCNC and 6G Summit, thus part of this paper was published in [17]. 
- We propose a hybrid area-based secrecy metric named Weighted Secrecy Coverage (WSC) as a combination of two other area-based secrecy metrics taken from $[16]$.

- We perform extensive simulations to establish positioning and power trends that allow for the WSC optimization problem to be simplified.

Furthermore, the main contributions exclusive to this paper are the following:

- We formulate the WSC optimization problem as three independent MultiArmed Bandit problems, one for each positioning variable with Upper Confidence Bound (UCB) as the learning policy of choice.

- We establish a time frame-based approach with central processing in which a single frame, which we name a Secrecy Improvement Block (SIB), contains multiple Reinforcement Learning slots of central processing and a single Positioning slot in which the UAVs reallocate themselves based on a greedy policy.

- We write down an algorithm for the positioning of the UAVs to optimize the WSC of the system over time with the SIB approach.

- We run extensive simulations over a large number of experiments to obtain insight into the performance of the algorithm in terms of WSC values obtained and energy consumed over time for different simulation parameters.

- We also run simulations to assess the convergence of the algorithm over different parameters, and suggest convexity for the proposed optimization problem.

Notation: Throughout this paper, and unless stated otherwise, $|\cdot|$ denotes absolute value, $\mathbb{E}_{x}[\cdot]$ denotes the expectation over random variable $x$, if $x$ is missing the argument is considered the random variable, $\mathcal{N}\left(x, \sigma^{2}\right)$ denotes a normal distribution of mean $x$ and variance $\sigma^{2}, \operatorname{Pr}[\cdot]$ denotes probability, $F_{x}(\cdot)$ is the Cumulative Density Function (CDF) of random variable $x$ and $\iint_{S}$ is a double integral over surface $S$.

\section{Methods}

\subsection{System model}

Consider the system illustrated in Fig. 1, comprised of a legitimate pair of ground nodes, the transmitter Alice (A) and the receiver Bob (B), which are confined on a circular area $\mathrm{S}$ of radius $R_{A}$ around Alice. Within $\mathrm{S}$, we establish the presence of an illegitimate node Eve (E) that is trying to leak the information from the legitimate transmission. A is located at the origin of coordinates $(0,0,0)$ and Bob is located along the $\mathrm{x}$ axis at $\left(d_{\mathrm{AB}}, 0,0\right)$, without losing generality. The position of $\mathrm{E}$ remains unknown. To improve the secrecy performance of the system, two UAVs, $J_{1}$ and $\mathrm{J}_{2}$, are deployed to act as jammers by emitting pseudorandom noise isotropically in order to prevent $\mathrm{E}$ from leaking information. The jammers are positioned at a common height $h_{\mathrm{J}}$ and within a circular orbit of radius $R_{\mathrm{J}}$ around $\mathrm{A}$, at angular positions $\theta_{\mathrm{J}_{1}}$ and $\theta_{\mathrm{J}_{2}}$. We assume that the estimate of the radial position of $\mathrm{B}$ with respect to $\mathrm{A}$ is unreliable, thus we model the distance between $\mathrm{A}$ and $\mathrm{B}$ as a random Gaussian variable with the actual distance $d_{\mathrm{AB}}$ being the mean of the estimate (unbiased), and a given uncertainty $\sigma_{\mathrm{AB}}, \widehat{d}_{\mathrm{AB}} \sim \mathcal{N}\left(d_{\mathrm{AB}}, \sigma_{\mathrm{AB}}^{2}\right)$, where $\widehat{d}_{\mathrm{AB}}$ is the estimate of the distance between $\mathrm{A}$ and $\mathrm{B}$. 


\subsubsection{Ground channels}

There are two ground channels to consider between ground nodes, one between Alice an Bob, and the other between Alice and Eve. Both channels are considered to undergo Rayleigh fading and are subject to additive white Gaussian noise (AWGN) with mean power $N_{0}$. Then the corresponding channel coefficients are $\mathrm{h}_{\mathrm{AB}}$ and $\mathrm{h}_{\mathrm{AE}}$, and the respective channel gains are $g_{\mathrm{AB}}=\left|\mathrm{h}_{\mathrm{AB}}\right|^{2}$ and $g_{\mathrm{AE}}=\left|\mathrm{h}_{\mathrm{AE}}\right|^{2}$. For a node $U \in\{B, E\}$, the channel coefficient $\mathrm{h}_{\mathrm{AU}}$ is an independent complex circularlysymmetric Gaussian random variable with variance $\mathbb{E}\left[\left|\mathrm{h}_{\mathrm{AU}}\right|^{2}\right]=\mathbb{E}\left[g_{\mathrm{AU}}\right]=d_{\mathrm{AU}}^{-\alpha_{G}}$, where $d_{\mathrm{AU}}$ is the distance between $\mathrm{A}$ and node $\mathrm{U}$ and $\alpha_{G}$ is the pathloss exponent for the ground links. Then, the channel gain $g_{\mathrm{AU}}$ follows an exponential distribution with parameter $\Omega_{\mathrm{AU}}=d_{\mathrm{AU}}^{\alpha_{G}}$.

\subsubsection{Air-to-ground channels}

There are two air-to-ground channels for each UAV jammer, one between the UAV and $\mathrm{B}$, and the other between the UAV and E. The channel coefficients and gains for those links are, respectively, given by $\mathrm{h}_{\mathrm{J}_{\mathrm{i}} \mathrm{U}}$ and $g_{\mathrm{J}_{\mathrm{i}} \mathrm{U}}=\left|\mathrm{h}_{\mathrm{J}_{\mathrm{i}} \mathrm{U}}\right|^{2}$ with $U \in\{B, E\}$ for both ground nodes and $i \in\{1,2\}$ for both UAVs. These channels are considered deterministic and have a contribution from a LoS component and a Non-LoS (NLoS) component, where the contribution of each component to the overall channel are given by probabilities $P_{\mathrm{LoS}}$ and $P_{\mathrm{NLoS}}$ respectively [19]. These probabilities are a function of the UAV position with respect to the ground node of interest $\mathrm{U}$, and are given by [19]

$$
\begin{aligned}
P_{\mathrm{LoS}} & =\frac{1}{1+\psi \exp \left(-\omega\left[\frac{180}{\pi} \tan ^{-1}\left(\frac{h_{\mathrm{J}}}{r_{\mathrm{J}_{\mathrm{i}} \mathrm{U}}}\right)-\psi\right]\right)}, \\
P_{\mathrm{NLoS}} & =1-P_{\mathrm{LoS}}
\end{aligned}
$$

where $\psi$ and $\omega$ are environmental constants $[20,21]$ and $r_{\mathrm{J}_{\mathrm{i}} \mathrm{U}}$ is the distance from node $\mathrm{U}$ and the projection on the plane of UAV $i$.

Then, the average pathloss of the air-to-ground channels, between UAV $i$ and ground node $U$ is given by

$$
\begin{aligned}
L_{\mathrm{J}_{\mathrm{i}} \mathrm{U}} & =P_{\mathrm{LoS}} \eta_{\mathrm{LoS}} d_{\mathrm{J}_{\mathrm{i}} \mathrm{U}}^{\alpha_{J}}+P_{\mathrm{NLoS}} \eta_{\mathrm{NLoS}} d_{\mathrm{J}_{\mathrm{i}} \mathrm{U}}^{\alpha_{J}}, \\
& =\left(h_{\mathrm{J}}^{2}+r_{\mathrm{J}_{\mathrm{i}} \mathrm{U}}^{2}\right)^{\frac{\alpha_{J}}{2}}\left(P_{\mathrm{LoS}} \eta_{\mathrm{LoS}}+P_{\mathrm{NLoS}} \eta_{\mathrm{NLoS}}\right),
\end{aligned}
$$

where $\alpha_{J}$ is the pathloss exponent for the air-to-ground links and $\eta_{\mathrm{LoS}}$ and $\eta_{\mathrm{NLoS}}$ are the attenuation factors for the LoS and the NLoS links, respectively. Thus, the air-to-ground channel coefficients are expressed as $\mathrm{h}_{\mathrm{J}_{\mathrm{i}} \mathrm{U}}=\left(\sqrt{L_{\mathrm{J}_{\mathrm{i}} \mathrm{U}}}\right)^{-1}$.

\subsubsection{Signal analysis}

For the communication process, A sends a symbol $x[m]$ with mean power $\mathbb{E}\left[|x[m]|^{2}\right]=P_{\mathrm{A}}$ at time $m$ while the UAVs send pseudo-random symbols $s_{i}[m]$ with mean power $\mathbb{E}\left[\left|s_{i}[m]\right|^{2}\right]=P_{\mathrm{J}_{\mathrm{i}}}$, with $i \in\{1,2\}$ for both UAVs. Considering a common noise level, such that noise samples $w[m]$ have power $\mathbb{E}\left[|w[m]|^{2}\right]=N_{0}$ at every node in the system, the transmit signal-to-noise ratio (SNR) at A and at the 
UAVs is given by $\gamma_{\mathrm{A}}=P_{\mathrm{A}} / N_{0}$ and $\gamma_{\mathrm{J}_{\mathrm{i}}}=P_{\mathrm{J}_{\mathrm{i}}} N_{0}$, respectively. Thus, the received signal at both $\mathrm{B}$ and $\mathrm{E}$ at time $m$ are respectively given by

$$
\begin{aligned}
& y_{\mathrm{B}}[m]=\mathrm{h}_{\mathrm{AB}} x[m]+\mathrm{h}_{\mathrm{J}_{1} \mathrm{~B}} s_{1}[m]+\mathrm{h}_{\mathrm{J}_{2} \mathrm{~B}} s_{2}[m]+w[m], \\
& y_{\mathrm{E}}[m]=\mathrm{h}_{\mathrm{AB}} x[m]+\mathrm{h}_{\mathrm{J}_{1} \mathrm{E}} s_{1}[m]+\mathrm{h}_{\mathrm{J}_{2} \mathrm{E}} s_{2}[m]+w[m] .
\end{aligned}
$$

Then, the signal-to-interference-plus-noise ratio (SINR) at B and E are given by $\gamma_{\mathrm{B}}=a_{\mathrm{J}} g_{\mathrm{AB}}$ and $\gamma_{\mathrm{E}}=b_{\mathrm{J}} g_{\mathrm{AE}}$ where $a_{\mathrm{J}}$ and $b_{\mathrm{J}}$ are given by

$$
\begin{aligned}
a_{\mathrm{J}} & =\frac{\gamma_{\mathrm{A}}}{1+g_{\mathrm{J}_{1} \mathrm{~B}} \gamma_{\mathrm{J}_{1}}+g_{\mathrm{J}_{2} \mathrm{~B}} \gamma_{\mathrm{J}_{2}}}, \\
b_{\mathrm{J}} & =\frac{\gamma_{\mathrm{A}}}{1+g_{\mathrm{J}_{1} \mathrm{E}} \gamma_{\mathrm{J}_{1}}+g_{\mathrm{J}_{2} \mathrm{E}} \gamma_{\mathrm{J}_{2}}} .
\end{aligned}
$$

For the particular case with no UAV jammers, then the SINR values at $\mathrm{B}$ and $\mathrm{E}$ are, respectively, given by $\gamma_{\mathrm{B}}=a_{\mathrm{NJ}} g_{\mathrm{AB}}$ and $\gamma_{\mathrm{E}}=b_{\mathrm{NJ}} g_{\mathrm{AE}}$ where $a_{\mathrm{NJ}}=b_{\mathrm{NJ}}=\gamma_{\mathrm{A}}$.

\subsection{Performance Analysis}

As previously mentioned, $\mathrm{E}$ is located within a circular area $\mathrm{S}$ around $\mathrm{A}$, but no further knowledge on the exact position of $\mathrm{E}$ is assumed, i.e. $\mathrm{E}$ can be in whichever point inside S. Therefore, to evaluate the secrecy performance of the proposed system, we consider the area-based secrecy metrics proposed in [16], namely jamming coverage (JC) and jamming efficiency (JE), and a new hybrid metric, the WSC, introduced in [17]. These metrics' definition is based on the secrecy outage probability (SOP), which is derived for the proposed system as described below.

\subsubsection{Secrecy Outage Probability}

For the definition of the area-based secrecy metrics, we consider first the SOP [5] defined as:

$$
\mathrm{SOP}=\operatorname{Pr}\left[C_{S}<R_{S}\right]
$$

where $R_{S}$ is the chosen rate for a secrecy code and $C_{S}$ is the secrecy capacity, which for our system is given by:

$$
C_{S}=\left[C_{\mathrm{B}}-C_{\mathrm{E}}\right]^{+}=\left[\log _{2}\left(\frac{1+\gamma_{\mathrm{B}}}{1+\gamma_{\mathrm{E}}}\right)\right]^{+},
$$

where $C_{\mathrm{B}}$ and $C_{\mathrm{E}}$ are the capacities of the channels between Alice and Bob, and between Alice and Eve respectively, with $[X]^{+}=\max [X, 0]$, which tells us that if the capacity of the illegitimate channel is greater than the capacity of the legitimate channel, no secrecy can be achieved.

\subsubsection{Secrecy Improvement Metric}

This metric measures the improvement on the secrecy performance of the proposed system, which is measured by the SOP, attained by the introduction of the friendly 
jamming sent by the UAV jammers. Thus, this metric is given by [16]

$$
\Delta=\frac{\mathrm{SOP}_{\mathrm{NJ}}}{\mathrm{SOP}_{\mathrm{J}}}
$$

where the SOP subscript identifies if the SOP is computed with $(\mathrm{J})$ or without (NJ) the presence of friendly jamming. Then, $\Delta>1$ values imply a reduction on the SOP by the presence of the UAV jammers while $\Delta<1$ is the opposite.

For mathematical tractability purposes, in [17] we proposed an analogous secrecy improvement metric that provides the same general idea with the criteria of secrecy achievement $(1-\mathrm{SOP})$ instead of SOP, thus given by

$$
\bar{\Delta}=\frac{1-\mathrm{SOP}_{\mathrm{J}}}{1-\mathrm{SOP}_{\mathrm{NJ}}}
$$

This new secrecy improvement metric $\bar{\Delta}$ is obtained in closed form as in the following proposition.

Proposition 1 The secrecy improvement metric, $\bar{\Delta}$, for the proposed system, is given by [17]

$$
\bar{\Delta}=e^{\Omega_{\mathrm{AB}}\left(2^{R_{S}}-1\right)\left(\frac{1}{a_{\mathrm{J}}}-\frac{1}{a_{\mathrm{NJ}}}\right)}\left(\frac{2^{R_{S}}\left(\frac{\Omega_{\mathrm{AB}}}{\Omega_{\mathrm{AE}}}\right)+1}{2^{R_{S}}\left(\frac{\Omega_{\mathrm{AB}}}{\Omega_{\mathrm{AE}}}\right)\left(\frac{b_{\mathrm{J}}}{a_{\mathrm{J}}}\right)+1}\right),
$$

Proof Considering $R_{S}>0$, the SOP can be expressed as follows

$$
\begin{aligned}
\mathrm{SOP} & =\operatorname{Pr}\left[\log _{2}\left(\frac{1+\gamma_{\mathrm{B}}}{1+\gamma_{\mathrm{E}}}\right)<R_{S}\right] \\
& =\operatorname{Pr}\left[\log _{2}\left(\frac{1+a \cdot g_{\mathrm{AB}}}{1+b \cdot g_{\mathrm{AE}}}\right)<R_{S}\right] \\
& =\operatorname{Pr}\left[g_{\mathrm{AB}}<\frac{1}{a}\left(2^{R_{S}}\left(1+b \cdot g_{\mathrm{AE}}\right)-1\right)\right] .
\end{aligned}
$$

This probability is the definition of the cumulative distribution function (CDF) of $g_{\mathrm{AB}}$ averaged over $g_{\mathrm{AE}}$, both exponential random variables with parameter $\Omega_{\mathrm{AB}}$ and $\Omega_{\mathrm{AE}}$, respectively, thus the SOP can be obtained as

$$
\begin{aligned}
\mathrm{SOP} & =\mathrm{E}_{g_{\mathrm{AE}}}\left[F_{g_{\mathrm{AB}}}\left(\frac{1}{a}\left(2^{R_{S}}\left(1+b \cdot g_{\mathrm{AE}}\right)-1\right)\right)\right] \\
& =\mathrm{E}_{g_{\mathrm{AE}}}\left[1-e^{-\Omega_{\mathrm{AB}} \frac{1}{a}\left(2^{R_{S}}\left(1+b \cdot g_{\mathrm{AE}}\right)-1\right)}\right] \\
& =1-\int_{0}^{\infty} e^{-\Omega_{\mathrm{AB}} \frac{1}{a}\left(2^{R_{S}}(1+b \cdot x)-1\right)} f_{g_{\mathrm{AE}}}(x) d x \\
& =1-\int_{0}^{\infty} e^{-\Omega_{\mathrm{AB}} \frac{1}{a}\left(2^{R_{S}}(1+b \cdot x)-1\right)} \Omega_{\mathrm{AE}} e^{-x \Omega_{\mathrm{AE}}} d x \\
& =1-e^{-\frac{\Omega_{\mathrm{AB}}}{a}\left(2^{R_{S}}-1\right)}\left(\frac{1}{2^{R_{S}}\left(\frac{\Omega_{\mathrm{AB}}}{\Omega_{\mathrm{AE}}}\right)\left(\frac{b}{a}\right)+1}\right) .
\end{aligned}
$$


Then, the jamming improvement $\bar{\Delta}$ in (11) is obtained as

$$
\bar{\Delta}=e^{\Omega_{\mathrm{AB}}\left(2^{R_{S}}-1\right)\left(\frac{1}{a_{\mathrm{J}}}-\frac{1}{a_{\mathrm{NJ}}}\right)}\left(\frac{2^{R_{S}}\left(\frac{\Omega_{\mathrm{AB}}}{\Omega_{\mathrm{AE}}}\right)+1}{2^{R_{S}}\left(\frac{\Omega_{\mathrm{AB}}}{\Omega_{\mathrm{AE}}}\right)\left(\frac{b_{\mathrm{J}}}{a_{J}}\right)+1}\right) .
$$

Note that $\bar{\Delta}$ is defined for $\mathrm{E}$ being at a given point inside $\mathrm{S}$.

\subsubsection{Weighted Secrecy Coverage}

As mentioned before, we assume no knowledge on the position of $\mathrm{E}$, other than it is located inside the circular region $\mathrm{S}$ within a radius $R_{A}$ from Alice, so we analyze the secrecy performance of the proposed system in terms of the area-based metrics in [16], the jamming coverage (JC) and the jamming efficiency (JE). Both of these metrics give us the notion on the effect over the secrecy performance inside $\mathrm{S}$ by the presence of the UAV jammers.

For the JC, consider that $\mathrm{E}$ is located at a single point within the area $\mathrm{S}$, where a certain $\bar{\Delta}$ value can be calculated, and we are interested in such points that lead into a $\bar{\Delta}>1$ value. Then, the jamming secrecy coverage is the integral over the area where $\bar{\Delta}>1$, expressed as

$$
\mathrm{JC}=\iint_{\bar{\Delta}>1} d S_{\mathrm{E}}
$$

where the $d S_{\mathrm{E}}$ term indicates an integral over the positions of $\mathrm{E}$ over the whole area S. To illustrate this concept, Fig. 2 shows a simplified overview of the system as a heatmap of $\bar{\Delta}$ over the whole area S. The JC would be the total area where $\bar{\Delta}>1$, which is enclosed by the yellow line surrounding the UAVs and Alice.

On the other hand, JE measures the average improvement of the secrecy over the whole area $\mathrm{S}$ :

$$
\mathrm{JE}=\frac{1}{|S|} \iint_{S} \bar{\Delta} d S_{\mathrm{E}}
$$

where $|S|$ is the area of the region $\mathrm{S}$.

Note that, JC gives a measure of the area within $\mathrm{S}$ where an improvement on the secrecy performance of the system is obtained due to the UAV jammers, while JE gives a measure of the average improvement of the secrecy performance over the area $\mathrm{S}$, if $\mathrm{E}$ were located at a random point.

To get further insights on the jamming effective coverage, in [17] we proposed a hybrid metric, the WSC, to account for both, the area over which secrecy is improved and the average secrecy improvement over the whole area S. The WSC is given by

$$
\mathrm{WSC}=\left(\iint_{\bar{\Delta}>1} d S_{\mathrm{E}}\right)\left(\frac{1}{|\mathrm{~S}|} \iint_{\mathrm{S}} \bar{\Delta} d S_{\mathrm{E}}\right) .
$$




\subsection{Positioning Optimization}

In this section, we consider joint optimization of the $3 \mathrm{D}$ positioning of the UAVs (common height, common orbit radius and angles around A) and the power allocation between the UAVs in order to maximize the WSC, given a relative position of $\mathrm{B}$ with respect to $\mathrm{A}$, which is characterized by $d_{\mathrm{AB}}$. Thus, the optimization problem is formulated as

$$
\begin{array}{ll}
\max _{\Omega=\left\{\theta_{\mathrm{J}_{1}}, \theta_{\mathrm{J}_{2}}, \gamma_{\mathrm{J}_{1}} / \gamma_{\mathrm{J}_{2}}, h_{\mathrm{J}}, R_{\mathrm{J}}\right\}} & \operatorname{WSC}\left(\Omega, d_{\mathrm{AB}}\right) \\
\text { subject to } & 0 \leq \theta_{\mathrm{J}_{1}}, \theta_{\mathrm{J}_{2}} \leq 2 \pi, \\
& \gamma_{\mathrm{J}_{1}}, \gamma_{\mathrm{J}_{2}} \geq 0 \\
& \gamma_{\mathrm{J}_{1}}+\gamma_{\mathrm{J}_{2}} \leq \gamma_{\mathrm{T}}, \\
& 0 \leq h_{\mathrm{J}} \leq h_{\mathrm{MAX}}, \\
& 0 \leq R_{\mathrm{J}} \leq R_{\mathrm{MAX}},
\end{array}
$$

where $h_{\mathrm{MAX}}$ is the height limit of the UAVs, $R_{\mathrm{MAX}}$ is the limit of the orbit radius around $\mathrm{A}$, which is the radius of $\mathrm{S}$, and $\gamma_{\mathrm{T}}$ is the maximum jamming transmit $\mathrm{SNR}$ from both UAVs.

In the following, we present some positioning trends that allow us to simplify the optimization problem, and introduce our RL based approach to solve the simplified maximization problem.

\subsubsection{Preliminaries}

To simplify the optimization problem in (20), we look at some trends regarding the angular positioning and jamming SNR allocated to both UAVs, which were corroborated after exhaustive simulations and analysis of patterns.

In this sense, for a certain set of heights and distances between Alice and Bob, we ran an exhaustive search for the optimal angles for both UAVs, such that the maximum WSC value is achieved. This process was carried out for different values of orbit radius $R_{\mathrm{J}}$. The results are shown in Fig. 3, where the angles for both UAVs are plotted, for which the highest WSC value was obtained. The blue line represents the optimal angle for $\mathrm{J}_{1}$ opening clockwise, and the red line represents the optimal angle for $\mathrm{J}_{2}$ opening counterclockwise, both measured opening from the left of the $\mathrm{x}$ axis, being $\mathrm{B}$ at the right of the $\mathrm{x}$ axis. From those results, it can be observed two patterns. First, the angles do not go beyond $90^{\circ}$ in either direction, suggesting that the optimal position for UAVs is behind Alice with regards to Bob's position. Also, it is observed that the opening angles seem to occur symmetrically for both UAVs, opening at the same angles in different directions. Considering this, our problem in (20) can be simplified by considering a single opening angle $\theta_{\mathrm{J}}$ as shown in Fig. 4 , which is the opening angle between both UAVs and ranges between 0 and $\pi$ radians.

Regarding the power allocation, we run a simulation considering a fixed total transmit power available to be distributed between both UAVs, with different values of the power ratio between $J_{1}\left(\gamma_{J_{1}}\right)$ and $J_{2}\left(\gamma_{J_{2}}\right)$. In this graph, as we go to 0 to the left in the $\mathrm{x}$ axis, less power is allocated to $\mathrm{J}_{1}$, and more power is allocated to $\mathrm{J}_{2}$. On the other hand, as we go to 1 to the right, the total power is allocated more evenly between both UAVs, being 1 the point of equal power allocation and 
0 the point where all power is allocated to $J_{1}$ and none to $J_{2}$. The results of this search are shown in Fig. 5, where for each power allocation ratio, the WSC is computed and plotted. Five curves are shown, one for each different value for total allocated jamming power determined by $\gamma_{\mathrm{T}}$. The main take-away from this figure is that the WSC seems to be maximized by having an equal allocation of jamming power between both UAVs. Moreover, note that the presence of the UAV jammers improves the secrecy performance, which is getting better as more power is allocated for jamming. Under these observations, we reformulate the optimization problem in $(20)$ as

$$
\begin{array}{ll}
\max _{\Omega=\left\{\theta_{\mathrm{J}}, h_{\mathrm{J}}, R_{\mathrm{J}}\right\}} & W S C\left(\Omega, d_{\mathrm{AB}}\right) \\
\text { subject to } & 0 \leq \theta_{\mathrm{J}} \leq \pi, \\
& 0 \leq h_{\mathrm{J}} \leq h_{\mathrm{MAX}}, \\
& 0 \leq R_{\mathrm{J}} \leq R_{\mathrm{MAX}},
\end{array}
$$

where we have three optimization variables, the opening angle $\theta_{\mathrm{J}}$, UAV common height $h_{\mathrm{J}}$, and UAV surveillance orbit radius $R_{\mathrm{J}}$.

\subsubsection{Reinforcement Learning Based Positioning}

Given that the estimate of the distance from A to B is unreliable, the optimization problem in (21) cannot be reliably solved. To account for the stochastic nature of the estimate of the distance to $\mathrm{B}, d_{\mathrm{AB}}$, we consider a coordinate-descent based [22] iterative scheme to reliably solve the optimization problem in (21) by employing a RL approach to ascertain the optimum positioning for the UAVs around Alice. Particularly, we model this problem as a Multi-Armed Bandit (MAB) problem, by considering the discrete positioning variables values as the arms or actions, and the WSC reading obtained at each step as the values or rewards. In the following, we briefly introduce the basis of the MAB problem and some relevant RL concepts to help us explain our approach ${ }^{[2]}$.

Multi-Armed Bandit Problem: A MAB problem consists of an agent (bandit) which has to choose at each time step among a set of actions (arms) to obtain rewards. At each step, each chosen action provides a reward, which is a random variable with a given distribution per action. The goal of the agent is to maximize the reward obtained over the time, which could be understood as choosing the optimum action, which is the action with the highest expected reward, so-called exploitation. This is done by keeping estimates of each of the actions' expected rewards. Therefore, it is also of interest to keep learning more about other actions to refine the estimates for each of them, which is called exploration. The action chosen at each step is determined by a policy, which in part sets the exploration/exploitation balance to be taken. An illustrative example of this learning process can be seen in Fig. 6.

Considering the optimization problem in (21), we have three positioning variables, the opening angle of both UAVs behind Alice $\left(\theta_{\mathrm{J}}\right)$, the common height of the UAVs $\left(h_{\mathrm{J}}\right)$, and the orbit radius of the UAVs around Alice $\left(R_{\mathrm{J}}\right)$. Each variable is separated

${ }^{[2]}$ We refer interested readers on RL and the MAB problem to [23]. 
into its own RL process, independent of the other two. For each positioning variable, we define its possible actions as a range of values the variable can take, which are given by the constraints in (21), and a discretized number of actions per variable $\left(N_{\theta}, N_{h}, N_{R}\right)$. Each action of a variable has a reward distribution, which corresponds to the distribution of WSC values obtained by performing that action. The goal is to be able to estimate with high accuracy which of the actions has the greatest expected reward. At each step, one of the actions is chosen following a policy and the received WSC reward is processed to contribute for the estimation of the expected reward (WSC) for said action.

To simplify the computations, we perform three separated RL processes, one for each positional variable with its own action range discretization. The RL loops for each of the variables are to be repeated back to back, alternating between the variables as illustrated in Fig. 7.

Considering that for each RL step of a given positioning variable, an assumption needs to be made regarding the other two positioning variables. The natural way of choosing which value should be consider for the other two positioning variables is to choose them in a greedy fashion, i.e. choose the values for the other two positioning variables that are estimated thus far to be the ones that lead to the highest reward. This implies that for any of the positioning variables, the RL process being carried out is non-stationary since the values for the other positioning variables, which are considered as part of the environment, change during the process, thus changing the environment. To account for the non-stationarity of the RL processes, consider the following generic estimate update rule [23]:

$$
Q_{n+1}=Q_{n}+\alpha_{n}\left[R_{n}-Q_{n}\right]
$$

where $Q_{n}$ is a generic action reward estimate at time $n, R_{n}$ is the observed reward at time $n$ and $\alpha_{n}$ is so-called the step size at time $n$, which controls the contribution of the observed data to the estimate at time $n$. As we consider that all observed rewards will contribute evenly to the estimate, we set $\alpha_{n}=1 / n$. However, in a non-stationary environment, we may want to give a higher weight to the new observations over the past observations, so that the RL process would be more sensitive to the environmental changes. To accomplish this, we set $\alpha_{n}=\alpha$ for all $n$ values to be a constant, such that $0<\alpha<1$ [23].

Regarding the policy to be used, we consider three popular MAB policies [23]:

$\epsilon$-greedy: At each time step, the agent chooses a random non-greedy action with probability $\epsilon$ and the greedy action with probability $1-\epsilon$. The greater the $\epsilon$ value is, the more exploration is done, indiscriminate of the non-greedy action. The action choice rule is given by [23]

$$
A_{t}= \begin{cases}\arg \max _{a} Q_{t}(a) & \text { with } 1-\epsilon \text { probability } \\ \text { random non greedy action } & \text { with } \epsilon \text { probability }\end{cases}
$$

where $A_{t}$ is the action to be chosen at time $t$ and $Q_{t}(a)$ is the estimated value of action $a$ at time $t$. 
Initial values: The initial values for the estimated action values are set at a high level relative to the rewards expected, usually at a higher level than the maximum reward expected. In combination with an $\epsilon$-greedy approach, this forces all actions to become greedy from the very start, before updating their estimation closer to their actual values, thus, exploring all actions thoroughly at the beginning. This increases the incentive for exploration at the first steps, but it is not suitable for non-stationary environments. This action values initialization is expressed as [23]

$$
Q_{0}(a)=Q_{0} \quad, \quad \forall a
$$

where $Q_{0}$ is a common initial value for all actions $a$.

Upper Confidence Bound: The action chosen at each step is determined by both the estimated value of the action thus far (greedy) but also by how much the action has been chosen in the past. This rule is determined by [23]

$$
A_{t}=\underset{a}{\arg \max }\left[Q_{t}(a)+c \sqrt{\frac{\ln (t)}{N_{t}(a)}}\right]
$$

where $N_{t}(a)$ is the number of times the action $a$ has been chosen up to time $t$ and $c$ is a constant parameter that controls the degree of exploration. Then, with this policy a continuous exploration is performed as time goes on in favor of less chosen actions over time that is controlled by the $c$ constant, which has to be set depending on the desired degree of exploration, and the expected reward values.

In Fig. 8, we see the comparisons between $\epsilon$-greedy approaches, initial value approaches (with $\epsilon$-greedy of $\epsilon=0.3$ ) and Upper Confidence Bound (UCB) approaches for the average reward obtained per step, and the Mean Squared Error (MSE) of the actions expected rewards, which is the average square error made with respect to the WSC action values by considering the actual distance $d_{\mathrm{AB}}$, i.e. the real action values. This is done for a single RL process for a large number of experiments.

We will consider an off-policy approach, i.e. we will get to learn about the environment with a given policy, but we will take a physical action with a different policy. Then, we are interested on learning as much as possible about the non-stationary environment in the RL steps in order to reduce as much as possible the MSE, so that the policy to be used in the RL processes is the UCB policy with a exploration parameter $c$ that is chosen to allow a wide exploration and a better adaptation to the non-stationarity nature of the environment.

\subsubsection{Secrecy Improvement Block}

$\mathrm{RL}$ loops will be employed over the positional variables of the UAVs in order to iteratively reach the optimum values in a coordinate descent fashion [22]. This processing is performed at A that has a global understanding of the system, and it transmits the positional information to the UAVs for physical adjustment. However, the transmission frequency of positional information to the UAVs is a concern, since every time this information is received, the UAVs are compelled to adjust their position, thus entailing energy consumption. If this occurs after each RL step of each variable, the movement of the UAVs may be unnecessarily erratic (given the randomness of 
the estimate and the discretization level of the variable domains), consume a high amount of energy from the UAVs over time, and introduce a substantial amount of delay, given that Alice needs to receive an acknowledgment (ACK) from the UAVs alerting that the required new position has been assumed before starting another RL step.

Thus, we propose a time frame-based scheme that splits a given time range, which we name a Secrecy Improvement Block (SIB), into individual slots, namely RL slots and Positioning slots as shown in Fig. 9. A SIB comprises $n R L B$ consecutive RL slots, and a single positioning slot at the end of it. At the beginning of an RL slot, a $\widehat{d}_{\mathrm{AB}}$ estimate is obtained and used in the rest of the slot, where a single RL step is performed for each of the positioning variables $\left(\theta_{\mathrm{J}}, h_{\mathrm{J}}, R_{\mathrm{J}}\right)$, one after another. Each RL steps assumes a greedy positioning from the other variables.

For the duration of the RL slots, A performs internal processing of the RL steps, and at the positioning slot, A chooses the greedy actions from the three positioning variables and transmits this information to the UAVs. Then, the UAVs assume their new positions based on this information and send an ACK signal to Alice, which, upon reception, starts another SIB as shown in Fig. 10. Therefore, we define an off-policy scheme, where we employ a greedy policy at the positioning slots, and a UCB policy at the RL slots.

Given this approach, each UAV incurs in energy consumption at each positioning slot that is simply given by: the energy needed to receive the positioning instructions from Alice $\left(E_{R X}\right)$, the energy needed to maneuver to its new position $\left(E_{M o v}\right)$ and the energy needed to send an ACK back to Alice $\left(E_{A C K}\right)$. This energy term is given by:

$$
\begin{aligned}
E & =E_{R X}+E_{A C K}+E_{M o v} \\
& =E_{R X}+E_{A C K}+\Delta t_{v} P_{M o v},
\end{aligned}
$$

where $P_{M o v}$ is the power needed by the UAV to maneuver, and $\Delta t_{v}$ is the time it takes the UAV to perform this change in position. Assuming that the UAV changes its position by assuming its new angle, height and radius in that order, then $\Delta t_{v}$ is given by:

$$
\begin{aligned}
\Delta t_{v} & =\frac{1}{v_{\mathrm{J}}}\left(|\Delta s|+\left|\Delta h_{\mathrm{J}}\right|+\left|\Delta R_{\mathrm{J}}\right|\right) \\
& =\frac{1}{v_{\mathrm{J}}}\left(\frac{1}{2} R_{\mathrm{J}_{0}}\left|\Delta \theta_{\mathrm{J}}\right|+\left|\Delta h_{\mathrm{J}}\right|+\left|\Delta R_{\mathrm{J}}\right|\right),
\end{aligned}
$$

where $v_{\mathrm{J}}$ is the maneuvering speed of the UAV (assumed constant throughout the flight), $\Delta \theta_{\mathrm{J}}, \Delta h_{\mathrm{J}}$ and $\Delta R_{\mathrm{J}}$ are the angle, height and radius variations, and $R_{\mathrm{J}_{0}}$ is the initial UAV radius value.

\subsubsection{MAB-based WSC improvement UAV positioning algorithm}

The concepts defined so far have the main goal of establishing the optimal position for the two UAV jammers in order to maximize the WSC, while Alice sends out information to Bob over the wireless medium. In Algorithm 1, we present the process followed by the proposed algorithm, where the variables in brackets $\left(\left[\theta_{\mathrm{J}}\right],\left[h_{\mathrm{J}}\right],\left[R_{\mathrm{J}}\right]\right)$ represent the action values estimates array for each of the variables. 


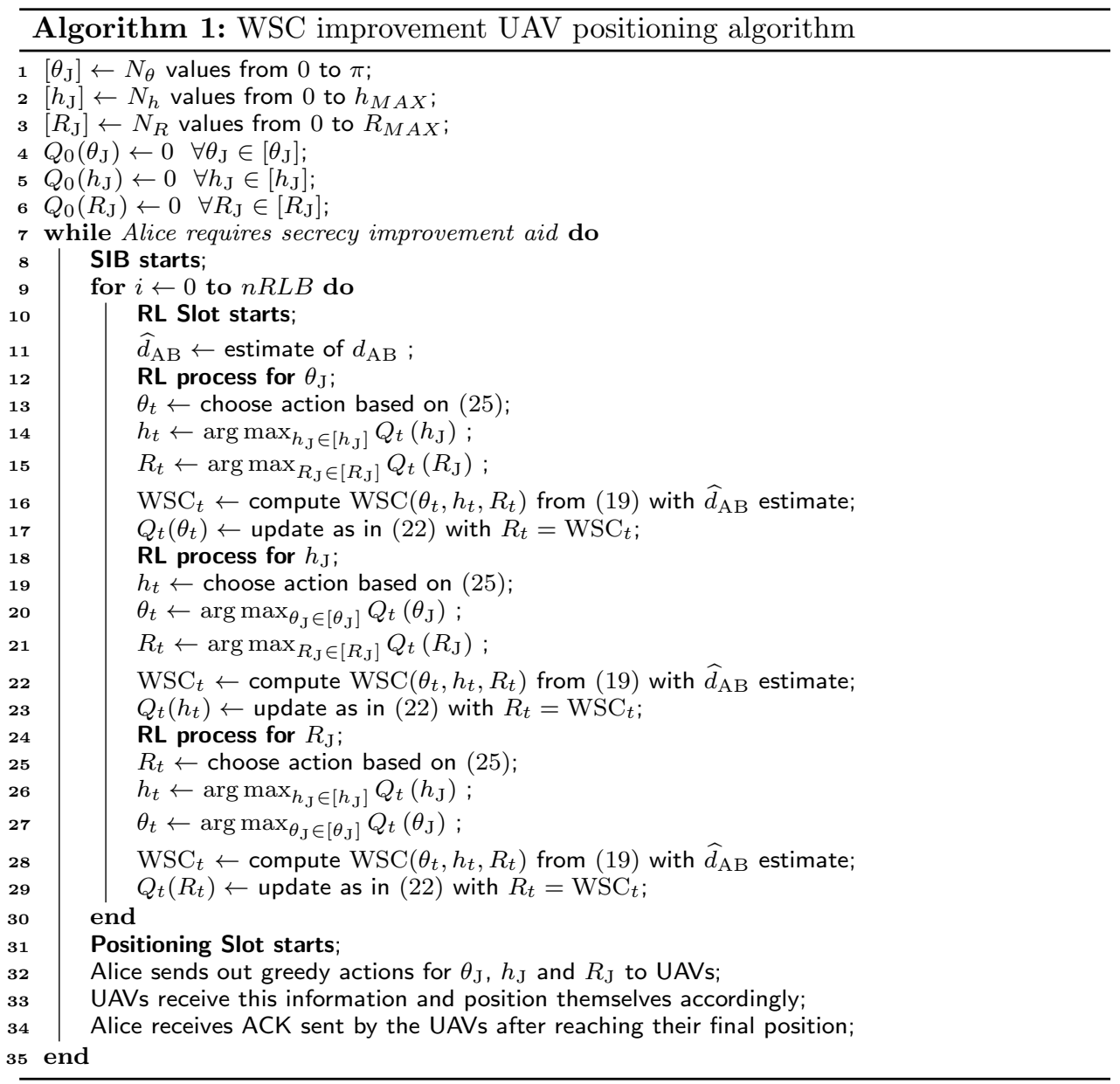

\section{Results and Discussion}

In this section, we evaluate the secrecy performance of the proposed system, in terms of the WSC, and the proposed RL algorithm for certain illustrative cases. The parameters used for the evaluation, unless stated otherwise, are shown in Table 1, channel specific parameters chosen for Urban environment taken from [20, 21]. For UAV specific parameters, such as the energy for receiving a data frame $E_{R X}$, for sending an ACK $E_{A C K}$, and the power spent on maneuvering from one point to another $P_{M o v}$, we refer to values based on common transceiver energy consumption values [24] and maneuvering power values [25]. Also, we consider a UAV movement speed of $v_{J}$ and a processing time for an RL slot of $\Delta t_{R L}$. The actual practical values of these parameters depend on the specific UAVs used, so the values considered are simplified for comparison purposes.

\subsection{Energy consumption and secrecy analysis}

In order to measure the performance of our algorithm we consider two performance metrics: the energy spent over time, and the secrecy performance in terms of the WSC obtained over time. In terms of energy, we will present it as a cumulative metric over a given time step, which will be at each SIB, or at each RL slot for the $n R L B$ comparison. The secrecy metric will be shown as the WSC, obtained at 
Table 1 Common simulation parameters

\begin{tabular}{|c|c|}
\hline Parameter & Value \\
\hline$\gamma_{\mathrm{A}}$ & 20 \\
\hline$\gamma_{\mathrm{T}}$ & 20 \\
\hline$d_{\mathrm{AB}}$ & $20[\mathrm{~m}]$ \\
\hline$R_{A}$ & $50[\mathrm{~m}]$ \\
\hline$\Delta t_{R L}$ & $0.3[\mathrm{~s}]$ \\
\hline$v_{J}$ & $2[\mathrm{~m} / \mathrm{s}]$ \\
\hline$E_{R X}$ & $0.1[\mathrm{~J}]$ \\
\hline$E_{A C K}$ & $0.1[\mathrm{~J}]$ \\
\hline$P_{M o v}$ & $80[\mathrm{~W}]$ \\
\hline$n S I B$ & 20 \\
\hline$n R L B$ & 20 \\
\hline$\sigma_{\mathrm{AB}}$ & $1[\mathrm{~m}]$ \\
\hline$c$ & 0.3 \\
\hline$\alpha$ & 0.5 \\
\hline$N_{\theta}$ & 90 \\
\hline$N_{h}$ & 30 \\
\hline$N_{R}$ & 30 \\
\hline$h_{\mathrm{MAX}}$ & $30[\mathrm{~m}]$ \\
\hline$R_{\mathrm{MAX}}$ & $50[\mathrm{~m}]$ \\
\hline$\psi($ Urban) & 9.61 \\
\hline$\omega$ (Urban) & 0.16 \\
\hline$\eta_{\text {LoS }}$ (Urban) & 1.0 \\
\hline$\eta_{\mathrm{NLoS}}($ Urban) & 20 \\
\hline$\alpha_{G}($ Urban) & 0.3 \\
\hline$\alpha J$ (Urban) & 0.3 \\
\hline & \\
\hline
\end{tabular}

each time step, normalized by the value of the secrecy area. All values obtained are averaged over a large enough number of experiments, each of which starts with a random realization of the positional variables.

Fig. 11 shows the normalized WSC mean values and the cumulative energy consumption values obtained over time (measured in SIBs ${ }^{[3]}$ ) for different values of step size $\alpha$, and Fig. 12 shows the normalized WSC mean values and the cumulative energy consumption values obtained over time (measured in SIBs) for different values of exploration weights $c$ of the UCB policy.

From these figures, note that a step size of $\alpha_{n}=1 / n$, i.e. an equivalent weighting for all steps, is enough to accommodate for the non-stationarity of the environment for a big enough $c$ exploration value for UCB. It also achieves low energy consumption over time, however a small $\alpha_{n}=\alpha$ constant step size also proves to achieve good performance regarding secrecy and energy consumption and is simpler to implement.

Furthermore, if the exploration value $c$ of the UCB policy is too low, exploration halts completely at a given point, after which the system becomes completely impervious to the non-stationary nature of the environment. This causes an early flat behavior regarding energy consumption due to the system exclusively presents a greedy behavior, which limits the energy consumption at the expense of a poor performance in terms of WSC, which presents a considerable gap with respect to higher $c$ values. On the other hand, higher $c$ values present similar performance in secrecy and energy consumption.

Fig. 13 shows the normalized WSC mean values and the cumulative energy consumption values obtained over time (measured in SIBs) for different values of uncertainty of Bob's distance to Alice, $\sigma_{\mathrm{AB}}$.

[3] The simulation time per SIB depends on the specific system running the simulations. 
The uncertainty of Bob's position has a direct impact on the amount of secrecy obtained. The higher the uncertainty around Bob's position, the smaller the average secrecy obtained, and the worse it converges. It has also a great impact into the energy consumption over time by the UAVs. This is because the greater the uncertainty becomes, the more poorly the positional variables converge, thus more positional updates are required, so the problem turns unstable. The more certain about Bob's position we are, the quicker the system converges to the optimum position, spending less energy in each Positioning slot.

Fig. 14 shows the cumulative energy consumption over time (measured over RL slots) for different number of RL slots per SIB $(n R L B)$ values. Fig 15 shows the normalized WSC mean values over time, both measured in SIBs and in RL slots for different values of number of RL slots per SIB $(n R L B)$.

In terms of energy consumption over time, note that the smaller the $n R L B$ value, the higher the energy consumption. This is because at smaller $n R L B$ values, the positional updates occur more often and are more unstable given the shorter learning periods. On the other hand, in terms of secrecy, the WSC increases as the values of $n R L B$ become higher in terms of SIBs, since there are more RL slots per SIB, which allows more learning before a SIB measurement. However, if the measure is done at each RL slot, all of the $n R L B$ curves present the same secrecy performance, which is expected because in all cases the learning processes are the same, but they are measured further apart for bigger $n R L B$ values.

\subsection{Convergence analysis}

We are also interested in how reliably our algorithm converges to a given value for each positional variable $\left(\theta_{\mathrm{J}}, h_{\mathrm{J}}\right.$ and $\left.R_{\mathrm{J}}\right)$ over the time, and the impact of different parameters. For this purpose, we will consider the variance of the given positional variable chosen or of the WSC obtained in a given time step.

From Fig 15, we observe that as the amount of RL blocks per SIB increases, the convergence of the WSC improves, i.e. by measuring the WSC at each positioning block. However, if the WSC is measured at each RL slot, the WSC convergence behavior is the same regardless of the $n R L B$ value, which is expected since the higher the $n R L B$ the longer the WSC measurement spans over a constant stream of RL slots.

Fig. 16 shows the variance of the estimates for the positional variables $\theta_{\mathrm{J}}, h_{\mathrm{J}}, R_{\mathrm{J}}$, and the obtained WSC over time (measured over SIBs) for different uncertainty values for Bob's distance to Alice $\sigma_{\mathrm{AB}}$ normalized by the actual distance $d_{\mathrm{AB}}$.

For a small enough uncertainty of the distance between Alice and Bob $\sigma_{\mathrm{AB}}$, compared to the actual distance $d_{\mathrm{AB}}$, it is clear that the variances of the values for the positional variables $\left(\theta_{\mathrm{J}}, h_{\mathrm{J}}, R_{\mathrm{J}}\right)$ quickly converge over time to a given lower bound, and this lower bound decreases as the uncertainty on Bob's position decreases, thus approaching zero as the uncertainty of Bob's position goes to zero.

This indicates that the system quickly converges upon a set of optimal positions for the UAVs as often as the uncertainty of Bob's position allows. Note that for lower uncertainty regarding Bob, the variance for the positional values and the WSC go to zero, indicating that the UAVs reliably reach the same optimum point, which suggests that this is a global maximum and that the optimization problem (21) is convex. 


\section{Conclusions}

This paper investigated the secrecy performance of a wireless wiretap system aided by two friendly jamming UAVs with no accurate knowledge of the exact position of an eavesdropper in terms of the area-based secrecy metric WSC. We proposed an RL-based approach to achieve the optimum positioning of both UAVs for a simplified optimization problem that maximizes the WSC, having an uncertainty about the position of the legitimate receiver. We formulated the RL-based algorithm as a set of three consecutive MAB problems with UCB policies, each defined over one of the three positioning variables for the UAVs (opening angle behind Alice, common height and orbit radius around Alice). We established a time frame-based algorithm that allows for the UAVs to update their positioning information after a number of RL steps for the three variables, thus allowing for more stable UAV movement and less energy expenditure. Finally we studied the effects of the uncertainty on the position of Bob, the learning parameters $c$ and $\alpha$ and the number of RL slots before a positioning update $n R L B$, on the secrecy obtained in terms of WSC, the energy consumed by the UAVs and the overall convergence of the algorithm.

In terms of the design parameters, it is clear that the more RL blocks considered into a SIB, the better performance in terms of energy consumption is achieved due to the more stable convergence and more sparse physical updates. However, this has to be weighted by how expediently we may want the UAVs to take more favorable positions with higher WSC values.

The proper selection of exploration and update parameters in the learning processes allows for the algorithm to adapt to the non-stationarity of the environment introduced by the coordinate-descent approach. This is shown in terms of a reducing variance of the estimates as time goes on. However, it may also accommodate other sources of non-stationarity like dynamics of the ground nodes.

Importantly, the proposed algorithm and the SIB time-frame structure can be explored for solving different problems in novel wireless communications networks that require periodic parameter updates to be learned over time in a non-stationary environment.

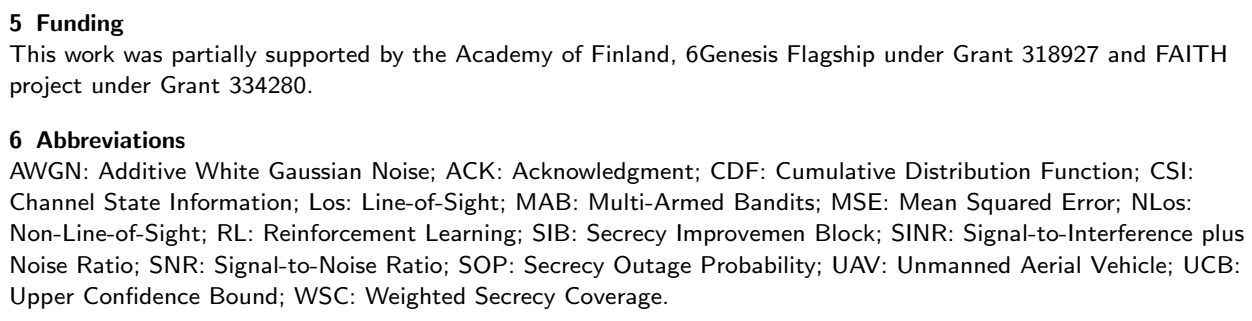


Author details

Centre for Wireless Communications, University of Oulu, Oulu, Finland.

\section{References}

1. Jiang, W., Han, B., Habibi, M.A., Schotten, H.D.: The road towards 6G: A comprehensive survey. IEEE Open Journal of the Communications Society 2, 334-366 (2021). doi:10.1109/OJCOMS.2021.3057679

2. Zeng, Y., Wu, Q., Zhang, R.: Accessing from the sky: A tutorial on UAV communications for $5 \mathrm{G}$ and beyond. Proceedings of the IEEE 107(12), 2327-2375 (2019). doi:10.1109/JPROC.2019.2952892

3. Porambage, P., Gür, G., Osorio, D.P.M., Liyanage, M., Gurtov, A., Ylianttila, M.: The roadmap to $6 \mathrm{G}$ security and privacy. IEEE Open Journal of the Communications Society 2, 1094-1122 (2021). doi:10.1109/OJCOMS.2021.3078081

4. Mucchi, L., Jayousi, S., Caputo, S., Panayirci, E., Shahabuddin, S., Bechtold, J., Morales, I., Stoica, R.-A., Abreu, G., Haas, H.: Physical-layer security in 6G networks. IEEE Open Journal of the Communications Society 2, 1901-1914 (2021). doi:10.1109/OJCOMS.2021.3103735

5. Moya Osorio, D.P., Vega Sanchez, J., Alves, H.: Physical-Layer Security for 5G and Beyond, pp. 1-19 (2019). doi:10.1002/9781119471509.w5GRef152

6. Sun, X., Ng, D.W.K., Ding, Z., Xu, Y., Zhong, Z.: Physical layer security in UAV systems: Challenges and opportunities. IEEE Wireless Communications 26(5), 40-47 (2019). doi:10.1109/MWC.001.1900028

7. Zhou, Y., Yeoh, P.L., Chen, H., Li, Y., Schober, R., Zhuo, L., Vucetic, B.: Improving physical layer security via a UAV friendly jammer for unknown eavesdropper location. IEEE Transactions on Vehicular Technology 67(11), 11280-11284 (2018). doi:10.1109/TVT.2018.2868944

8. Pang, X., Liu, M., Zhao, N., Chen, Y., Li, Y., Yu, F.R.: Secrecy analysis of UAV-based mmWave relaying networks. IEEE Transactions on Wireless Communications 20(8), 4990-5002 (2021). doi:10.1109/TWC.2021.3064365

9. Yapici, Y., Rupasinghe, N., Güvenç, I., Dai, H., Bhuyan, A.: Physical layer security for NOMA transmission in mmWave drone networks. IEEE Transactions on Vehicular Technology 70(4), 3568-3582 (2021). doi:10.1109/TVT.2021.3066350

10. Kim, M., Kim, S., Lee, J.: Securing communications with friendly unmanned aerial vehicle jammers. IEEE Transactions on Vehicular Technology 70(2), 1972-1977 (2021). doi:10.1109/TVT.2021.3052503

11. Nguyen, P.X., Nguyen, V.-D., Nguyen, H.V., Shin, O.-S.: UAV-assisted secure communications in terrestrial cognitive radio networks: Joint power control and 3D trajectory optimization. IEEE Transactions on Vehicular Technology 70(4), 3298-3313 (2021). doi:10.1109/TVT.2021.3062283

12. Wang, W., Li, X., Wang, R., Cumanan, K., Feng, W., Ding, Z., Dobre, O.A.: Robust 3D-trajectory and time switching optimization for dual-UAV-enabled secure communications. IEEE Journal on Selected Areas in Communications, 1-1 (2021). doi:10.1109/JSAC.2021.3088628

13. Guo, X., Chen, Y., Wang, Y.: Learning-based robust and secure transmission for reconfigurable intelligent surface aided millimeter wave UAV communications. IEEE Wireless Communications Letters 10(8), 1795-1799 (2021). doi:10.1109/LWC.2021.3081464

14. Dong, R., Wang, B., Cao, K.: Deep learning driven 3D robust beamforming for secure communication of UAV systems. IEEE Wireless Communications Letters 10(8), 1643-1647 (2021). doi:10.1109/LWC.2021.3075996

15. Zhang, Y., Mou, Z., Gao, F., Jiang, J., Ding, R., Han, Z.: UAV-enabled secure communications by multi-agent deep reinforcement learning. IEEE Transactions on Vehicular Technology 69(10), 11599-11611 (2020). doi:10.1109/TVT.2020.3014788

16. Vilela, J.P., Bloch, M., Barros, J., McLaughlin, S.W.: Wireless secrecy regions with friendly jamming. IEEE Trans. Inf. Forensics Security 6(2), 256-266 (2011). doi:10.1109/TIFS.2011.2111370

17. Cabezas, X.A.F., Osorio, D.P.M., Latva-aho, M.: Weighted secrecy coverage analysis and the impact of friendly jamming over UAV-enabled networks. In: 2021 Joint European Conference on Networks and Communications 6G Summit (EuCNC/6G Summit), pp. 124-129 (2021). doi:10.1109/EuCNC/6GSummit51104.2021.9482493

18. Cabezas, X.A.F., Osorio, D.P.M., Latva-aho, M.: Distributed UAV-enabled zero-forcing cooperative jamming scheme for safeguarding future wireless networks. In: 2021 IEEE International Symposium on Personal, Indoor and Mobile Radio Communications (PIMRC2021)

19. Zhou, Y., Yeoh, P.L., Chen, H., Li, Y., Schober, R., Zhuo, L., Vucetic, B.: Improving physical layer security via a UAV friendly jammer for unknown eavesdropper location. IEEE Trans. Veh. Technol. 67(11), 11280-11284 (2018). doi:10.1109/TVT.2018.2868944

20. Dao, V., Tran, H., Girs, S., Uhlemann, E.: Reliability and fairness for UAV communication based on non-orthogonal multiple access. In: 2019 IEEE International Conference on Communications Workshops (ICC Workshops), pp. 1-6 (2019). doi:10.1109/ICCW.2019.8757160

21. Al-Hourani, A., Kandeepan, S., Lardner, S.: Optimal LAP altitude for maximum coverage. IEEE Wireless Communications Letters 3(6), 569-572 (2014). doi:10.1109/LWC.2014.2342736

22. Wright, S.J.: Coordinate descent algorithms. Mathematical Programming 151(1), 3-34 (2015)

23. Sutton, R.S., Barto, A.G.: Reinforcement learning, second edition: An introduction. Adaptive Computation and Machine Learning series. MIT Press (2018). https://books.google.fi/books?id=uWVODwAAQBAJ

24. Moya Osorio, D.P., Benítez Olivo, E.E., Alves, H., Santos Filho, J.C.S., Latva-aho, M.: An adaptive transmission scheme for amplify-and-forward relaying networks. IEEE Transactions on Communications 65(1), 66-78 (2017). doi:10.1109/TCOMM.2016.2616136

25. Chan, C.W., Kam, T.Y.: A procedure for power consumption estimation of multi-rotor unmanned aerial vehicle. Journal of Physics: Conference Series 1509, 012015 (2020). doi:10.1088/1742-6596/1509/1/012015

10 Figures 
Figure 1 System model for the wireless wiretap channel within a secrecy area with two friendly UAV jammers.

Figure 2 Heatmap for $\bar{\Delta}$ values due to the presence of UAV jammers on a circular region around Alice.

Figure 3 Angles for both UAV jammers around Alice for which maximum WSC is achieved measured as a function of the radius of the jammers orbit around Alice $\left(d_{\mathrm{AB}}=20, \gamma_{\mathrm{A}}=15\right.$, $\gamma_{\mathrm{J}_{1}}=\gamma_{\mathrm{J}_{2}}=15$ ).

Figure 4 Opening angle simplification.

Figure 5 WSC achieved as a function of the ratio between transmit SNR of both UAV jammers for different values of total available jamming SNR $\gamma_{\mathrm{T}}\left(R_{\mathrm{J}}=28, d_{\mathrm{AB}}=20, \gamma_{\mathrm{A}}=\frac{1}{2} \gamma_{\mathrm{T}}\right.$, $\left.\gamma_{\mathrm{J}_{1}}+\gamma_{\mathrm{J}_{2}}=\frac{1}{2} \gamma_{\mathrm{T}}\right)$.

Figure 6 Reinforcement learning process: 1) At time $t$ the agent chooses an action $A_{t}$ based on a policy and the set of estimates $\left.\left\{Q_{a}\right\}, 2\right)$ the agent applies action $A_{t}$ onto the environment, 3) the agent obtains reward $R_{t}$ from the environment, and 4) the agent updates the estimate for the action chosen $Q_{A_{t}}$.

Figure 7 Reinforcement learning processes flow.

Figure 8 Comparison between policies in terms of (a) average reward obtained per step and (b) Mean Squared Error (MSE) per step. Both for UCB ( $c$ values), $\epsilon$-greedy ( $\epsilon$ values), and initial values $\left(Q_{0}\right.$ values).

Figure 9 Secrecy Improvement Block (SIB) structure with their internal structures and policies.

Figure 10 WSC improvement algorithm. (a) Iterative RL processes on the three positional variables $\theta_{\mathrm{J}}, h_{\mathrm{J}}$ and $R_{\mathrm{J}}$ in Alice, over the RL slots of the SIB, (b) signaling from Alice to the UAVs to adopt new positions, and (c) UAVs sending ACK signaling back to Alice after adopting their new positions.

Figure 11 (a) Normalized WSC mean values obtained over time, and (b) Cumulative energy consumed by a single UAV over time. Both measured over SIBs with varying step-size values for the RL updates $\alpha$.

Figure 12 (a) Normalized WSC mean values obtained over time, and (b) Cumulative energy consumed by a single UAV over time. Both measured over SIBs with varying exploration weights for the UCB policy $c$. 
Figure 13 (a) Normalized WSC mean values obtained over time, and (b) Cumulative energy consumed by a single UAV over time. Both measured over SIBs with varying uncertainty values for Bob's distance to Alice $\sigma_{\mathrm{AB}}$.

Figure 14 Cumulative energy consumed by a single UAV over time (measured over RL slots) with varying number of $\mathrm{RL}$ slots per $\mathrm{SIB}(n R L B)$ values.

Figure 15 Normalized WSC mean values obtained over time with varying number of RL slots per $\mathrm{SIB}(n R L B)$ values, measured over (a) SIBs, and (b) RL slots.

Figure 16 Estimate variance over time (measured over SIBs) with varying uncertainty values for Bob's distance to Alice $\sigma_{\mathrm{AB}}$ normalized by the actual distance $d_{\mathrm{AB}}$ for (a) the opening angle $\theta_{\mathrm{J}}$, (b) the common height $h_{\mathrm{J}}$, (c) the orbit radius $R_{\mathrm{J}}$, and (d) the normalized WSC. 


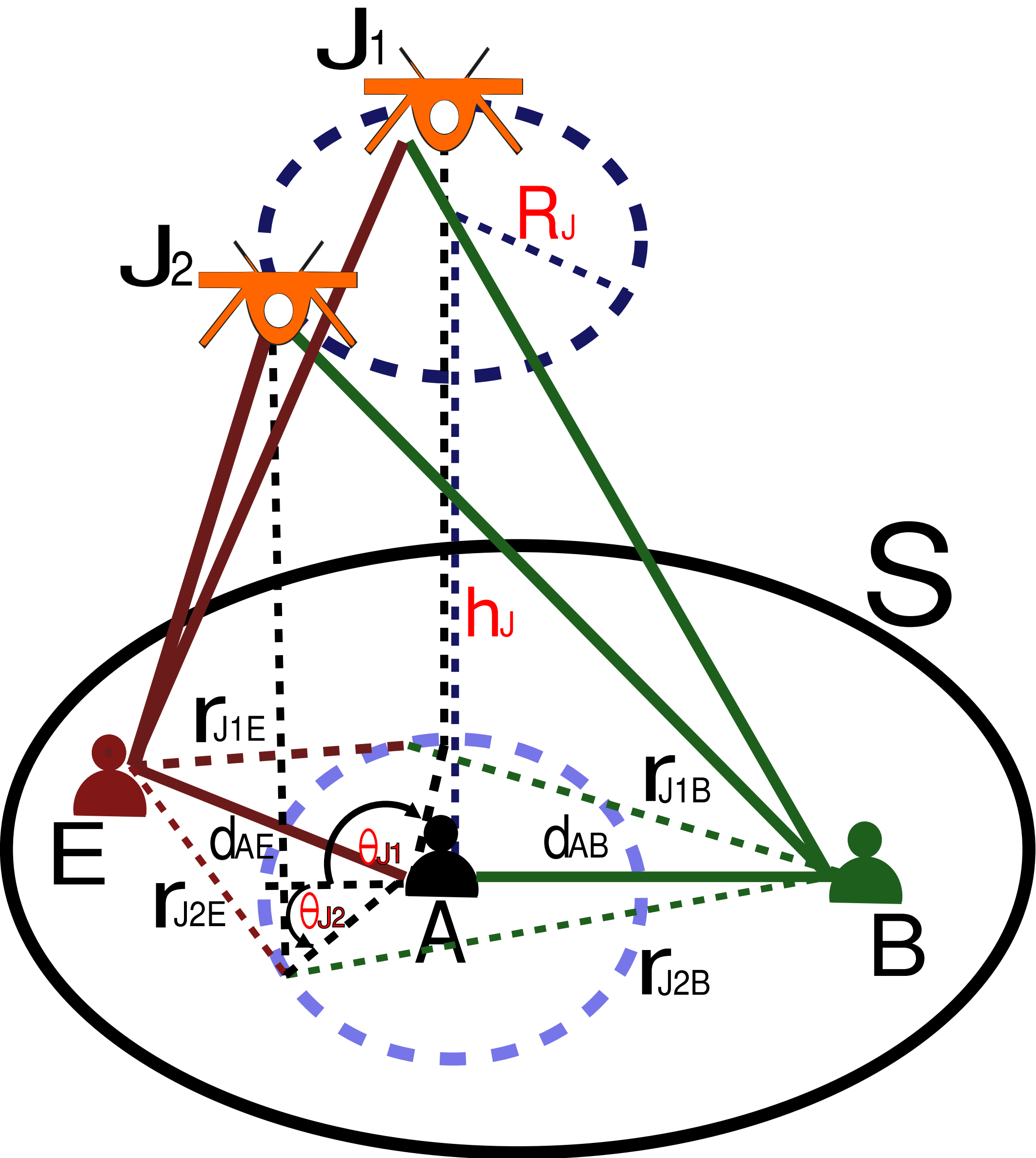




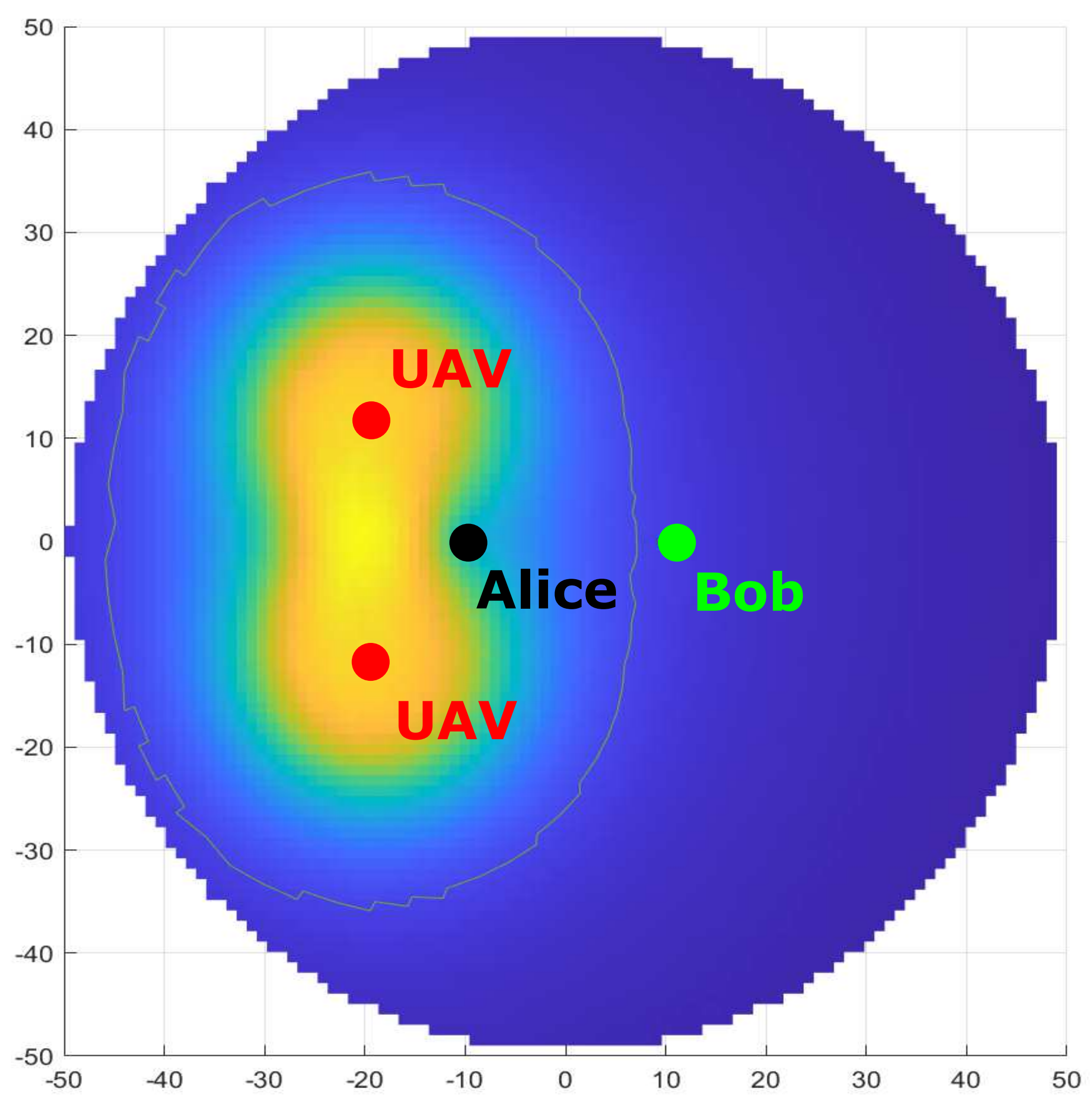




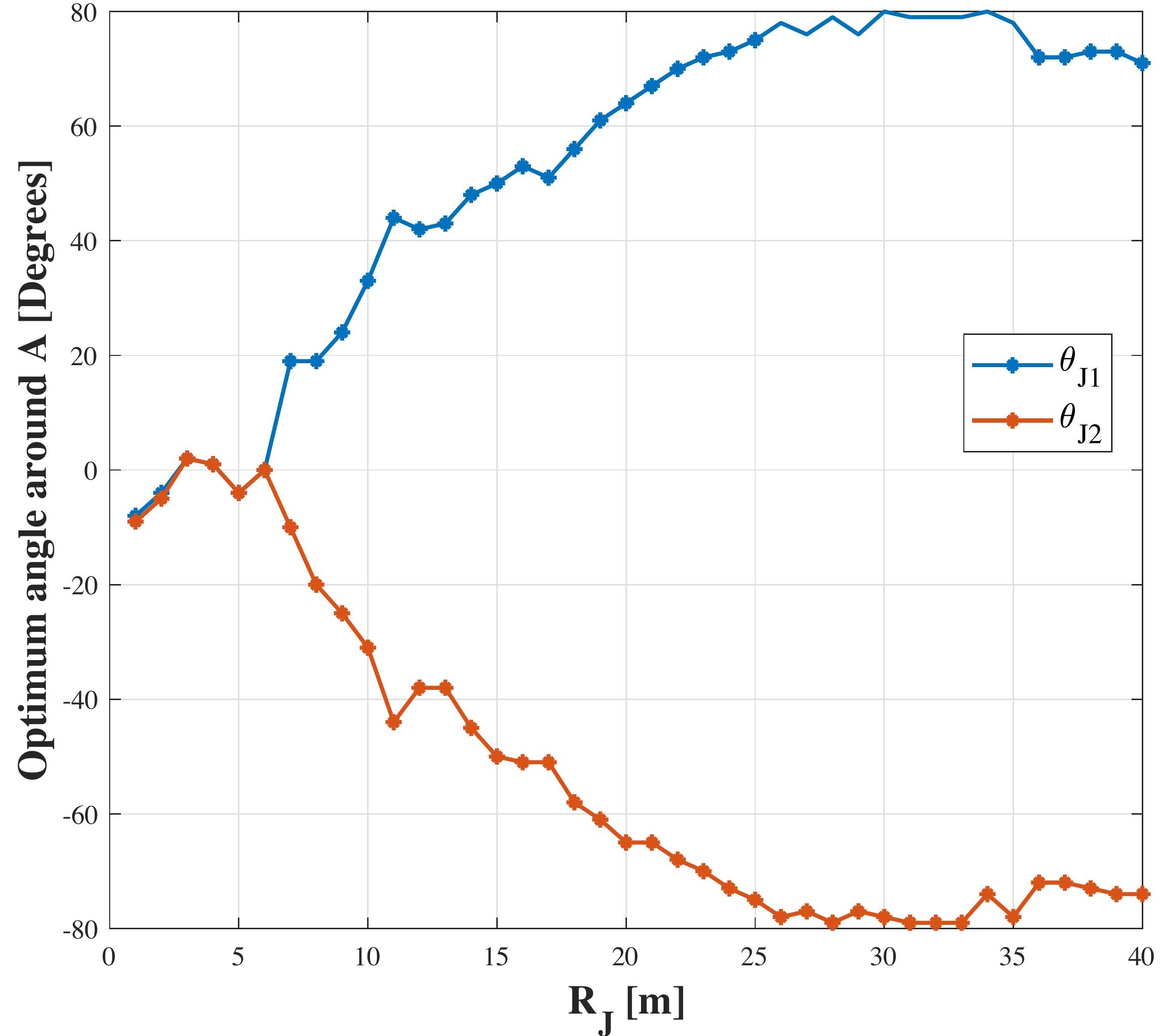




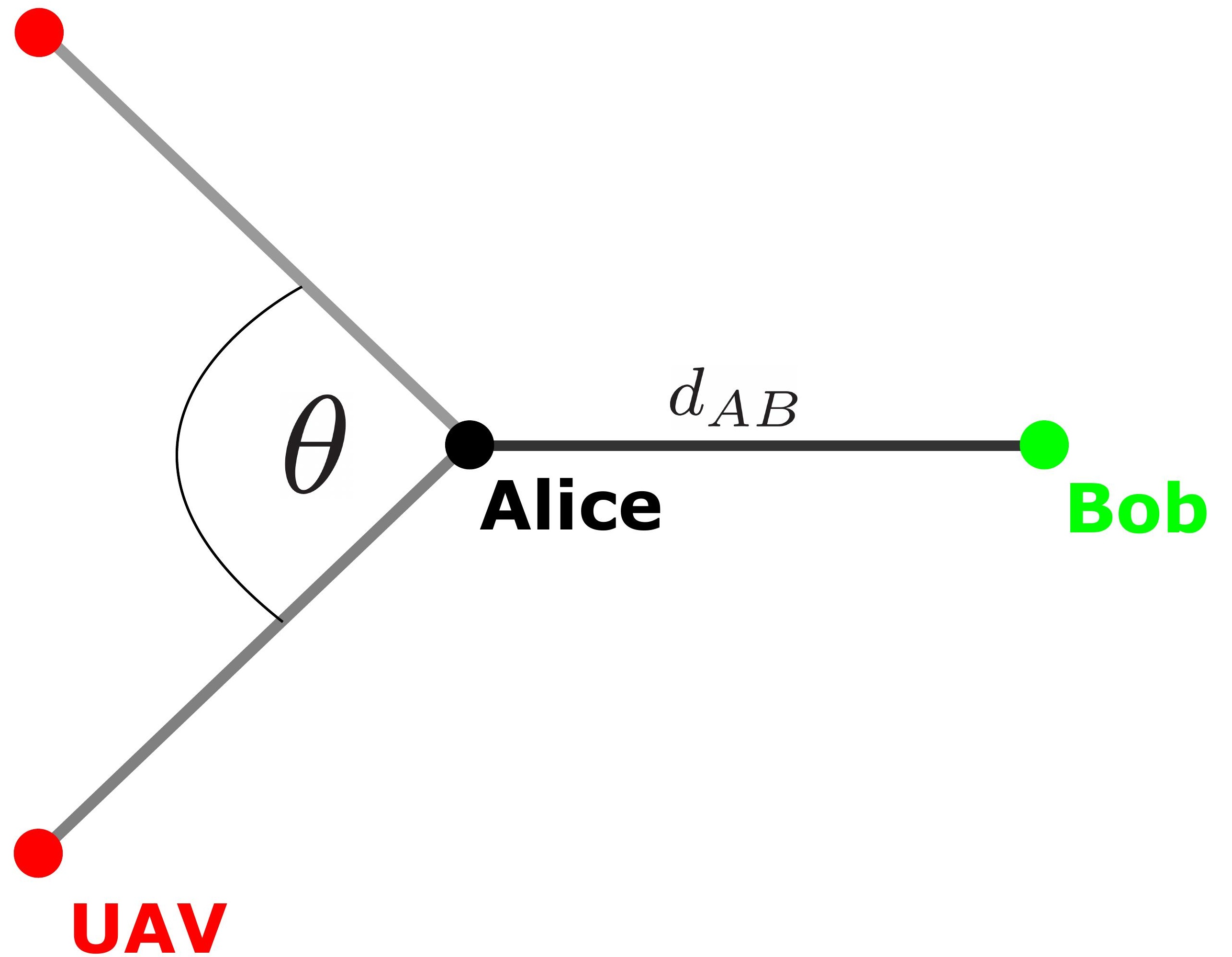









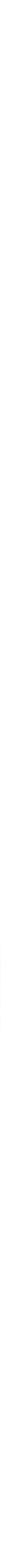




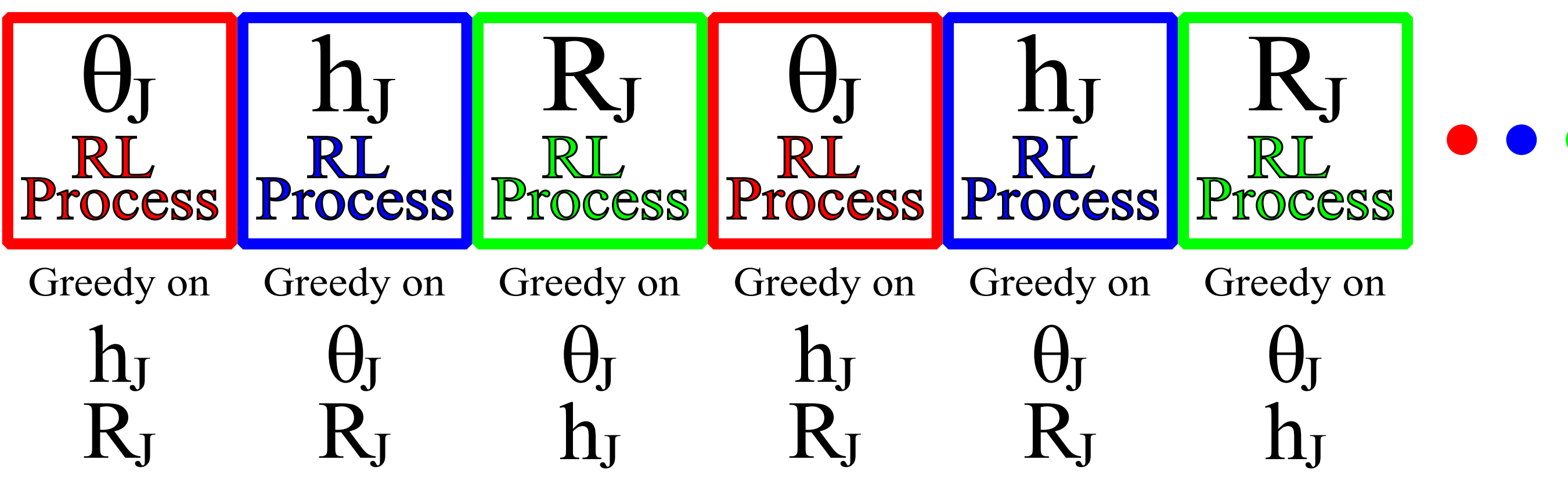


(a)



RL Slots (b)
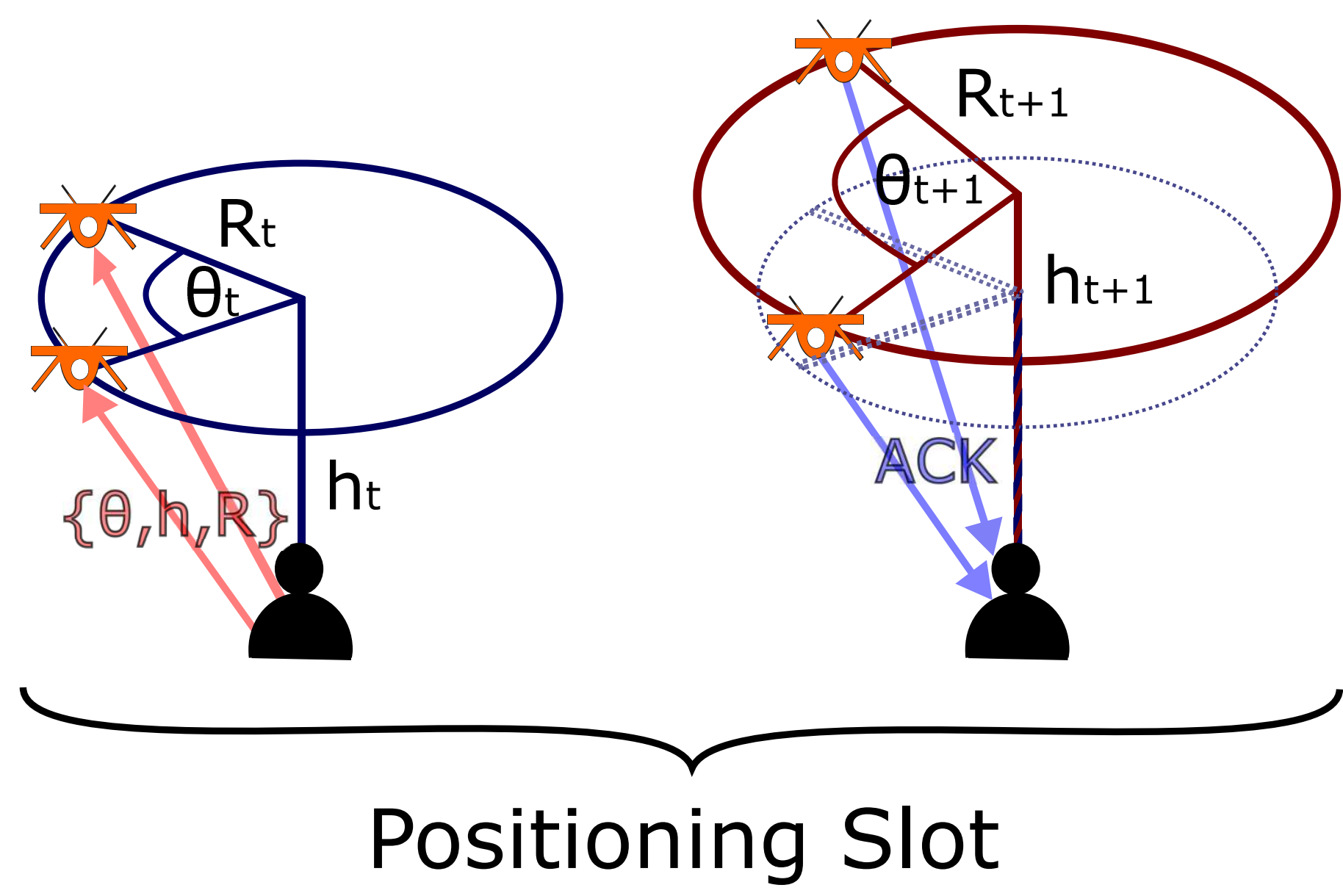

Positioning Slot

(c) 
(a)

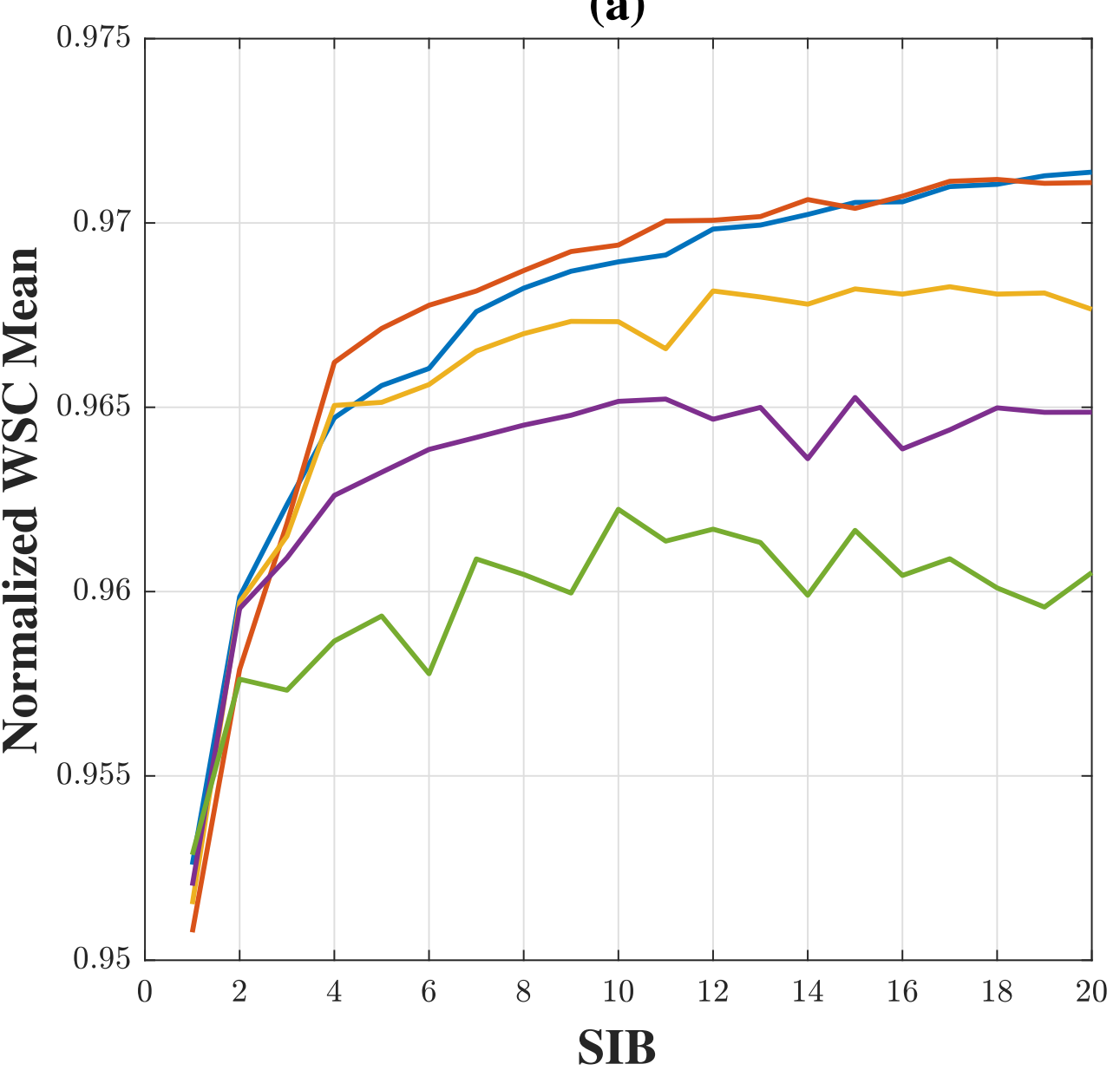

(b)

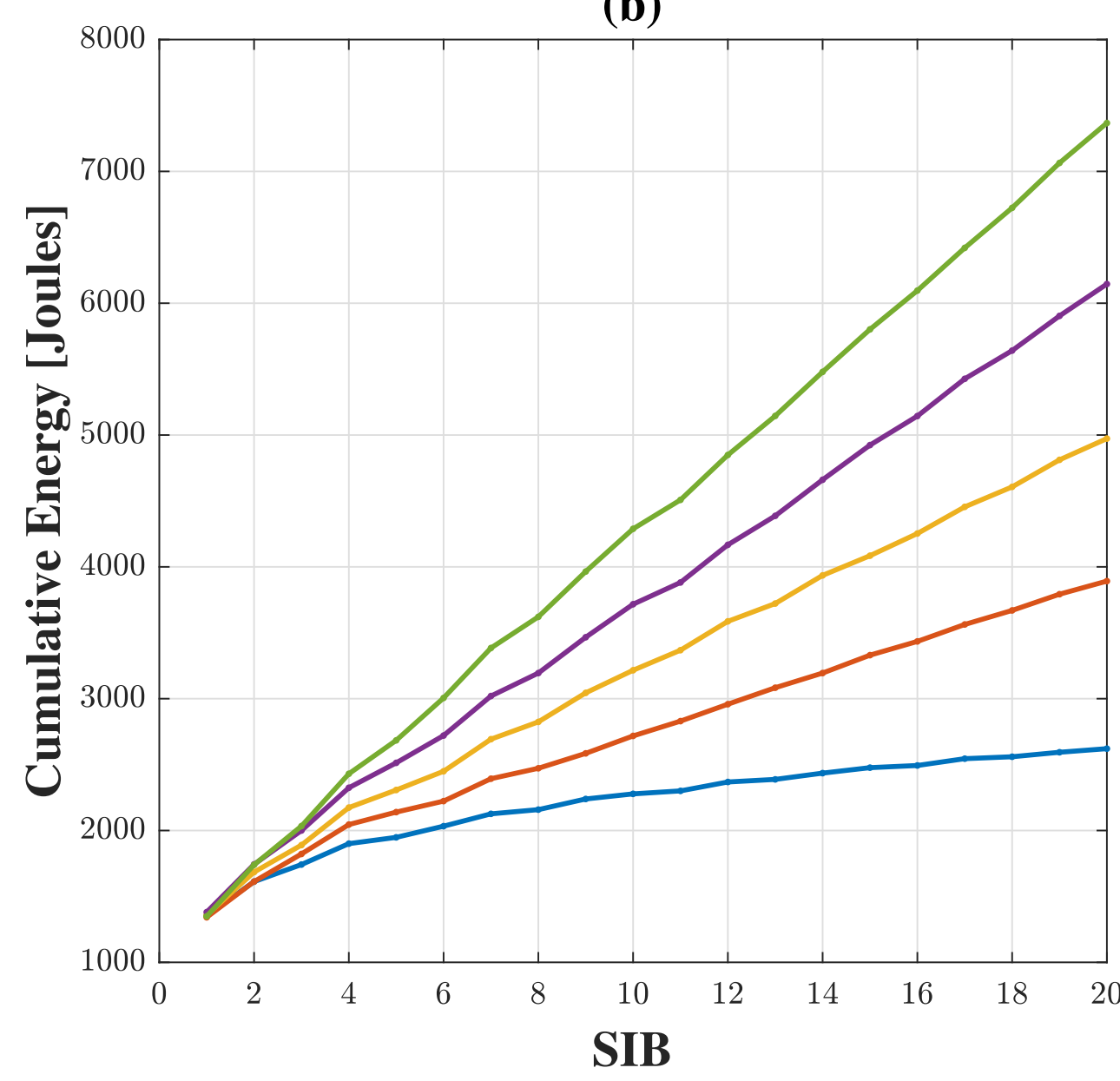

$-\alpha=1 / \mathrm{n}-\alpha=0.25$

$\alpha=0.5$

$\alpha=0.75$

$\alpha=0.99$ 
(a)

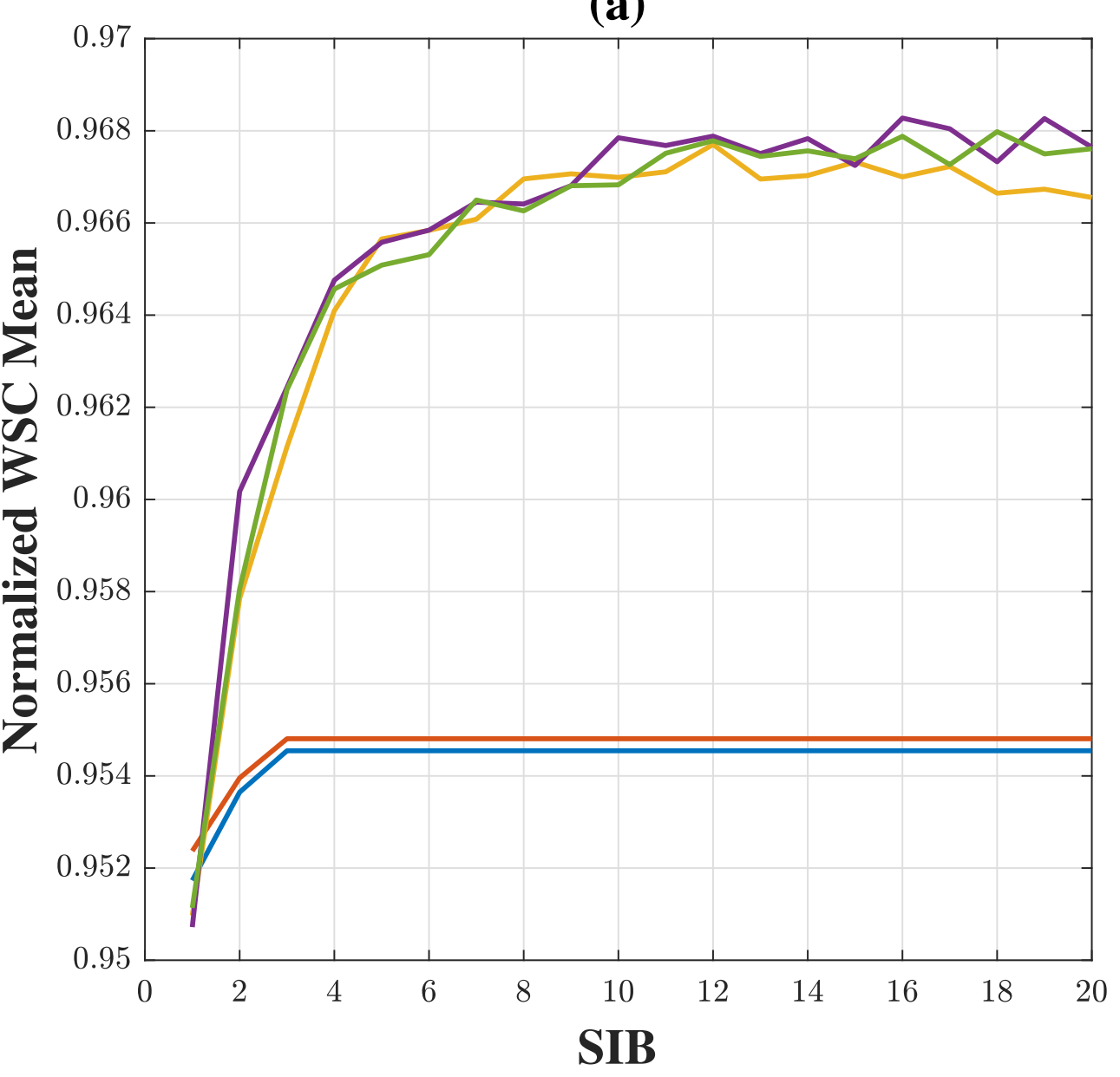

(b)

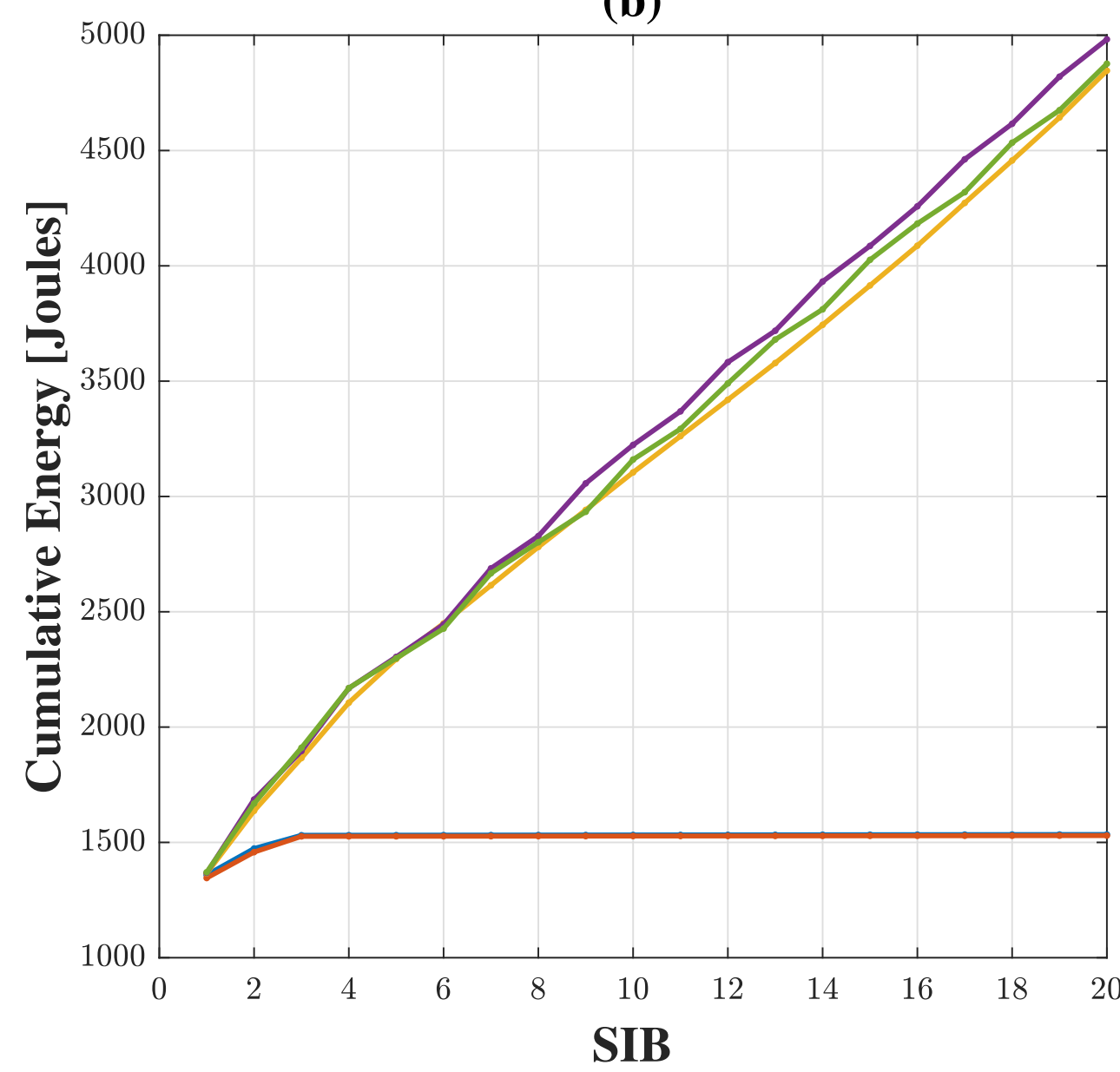

$$
\longrightarrow \mathrm{c}=0.001 \longrightarrow \mathrm{c}=0.01 \longrightarrow \mathrm{c}=0.1 \longrightarrow \mathrm{c}=0.3 \longrightarrow \mathrm{c}=0.5
$$


(a)



(b)

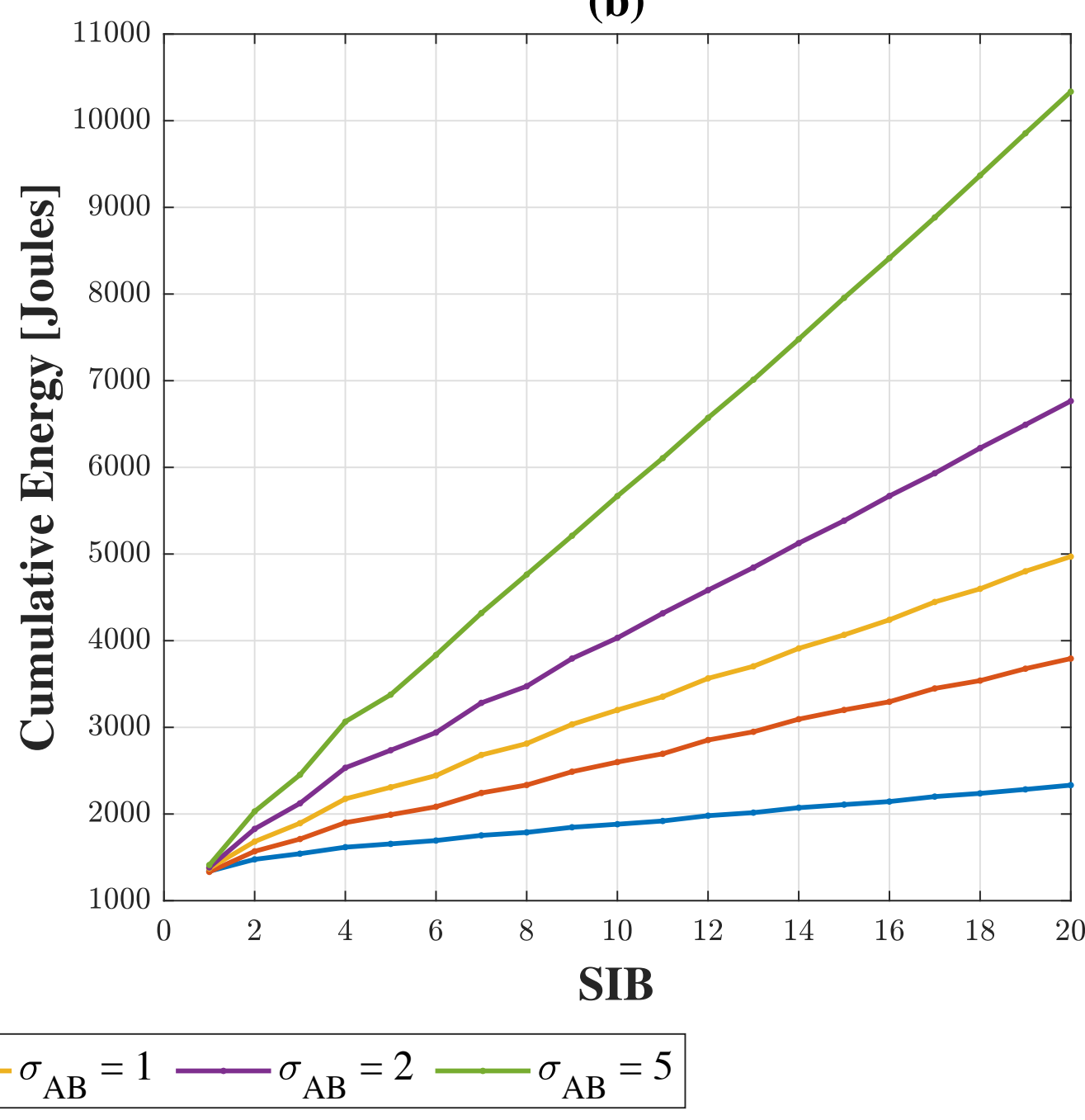




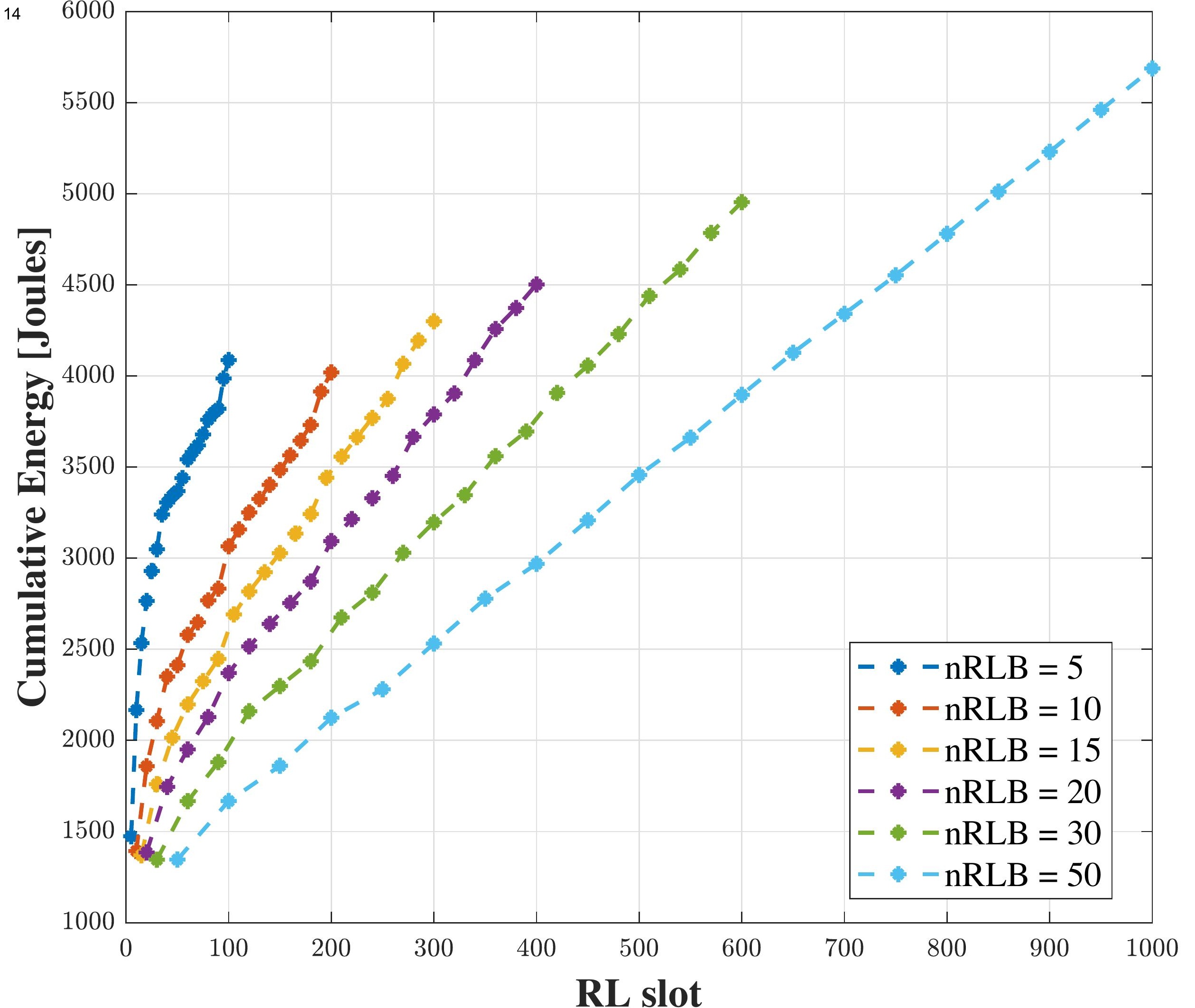


(a)



(b)

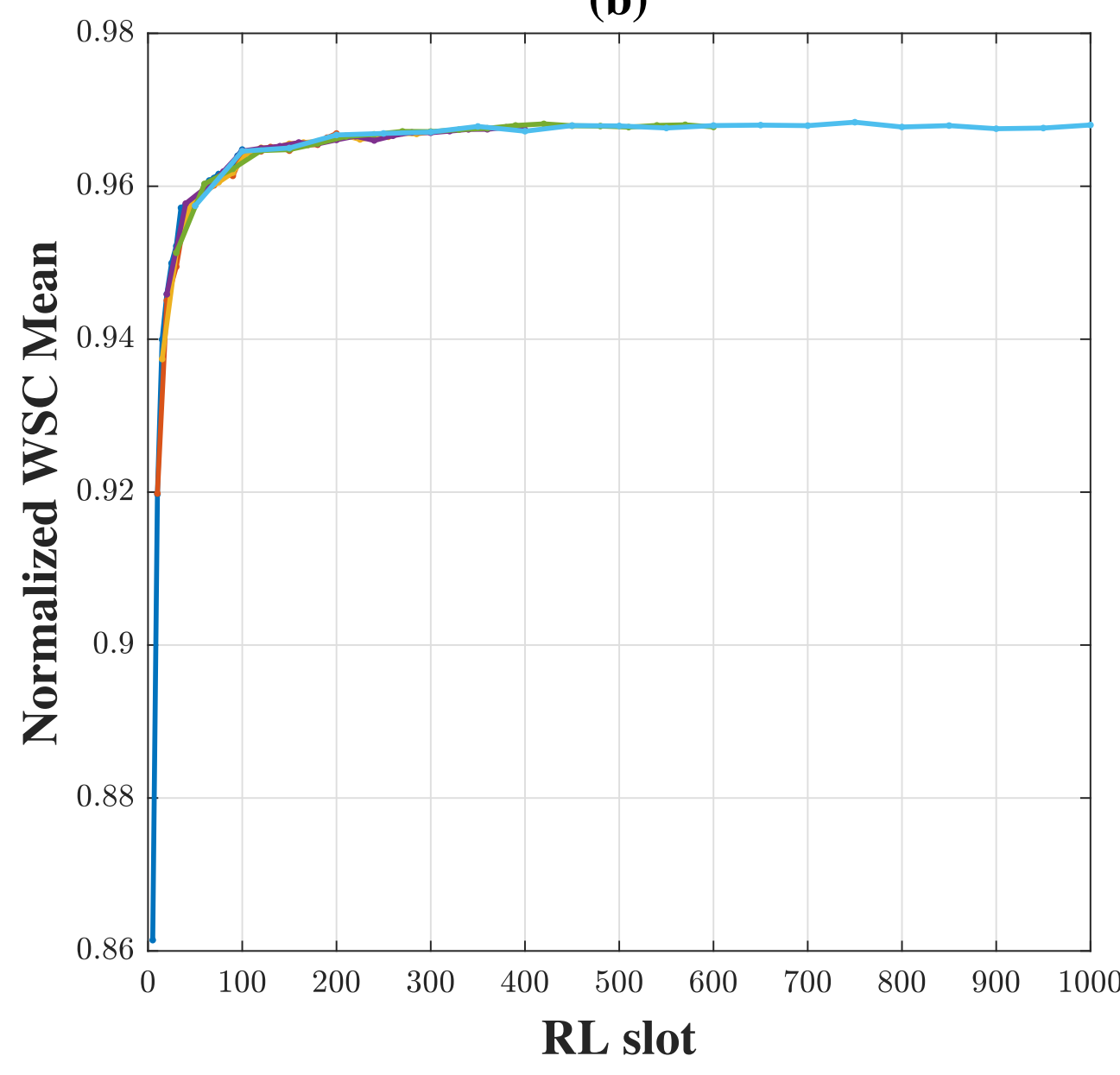

$\mathrm{nRLB}=5-\mathrm{nRLB}=10-\mathrm{nRLB}=15$

$\mathrm{nRLB}=20$

$\mathrm{nRLB}=30$

$\mathrm{nRLB}=50$ 

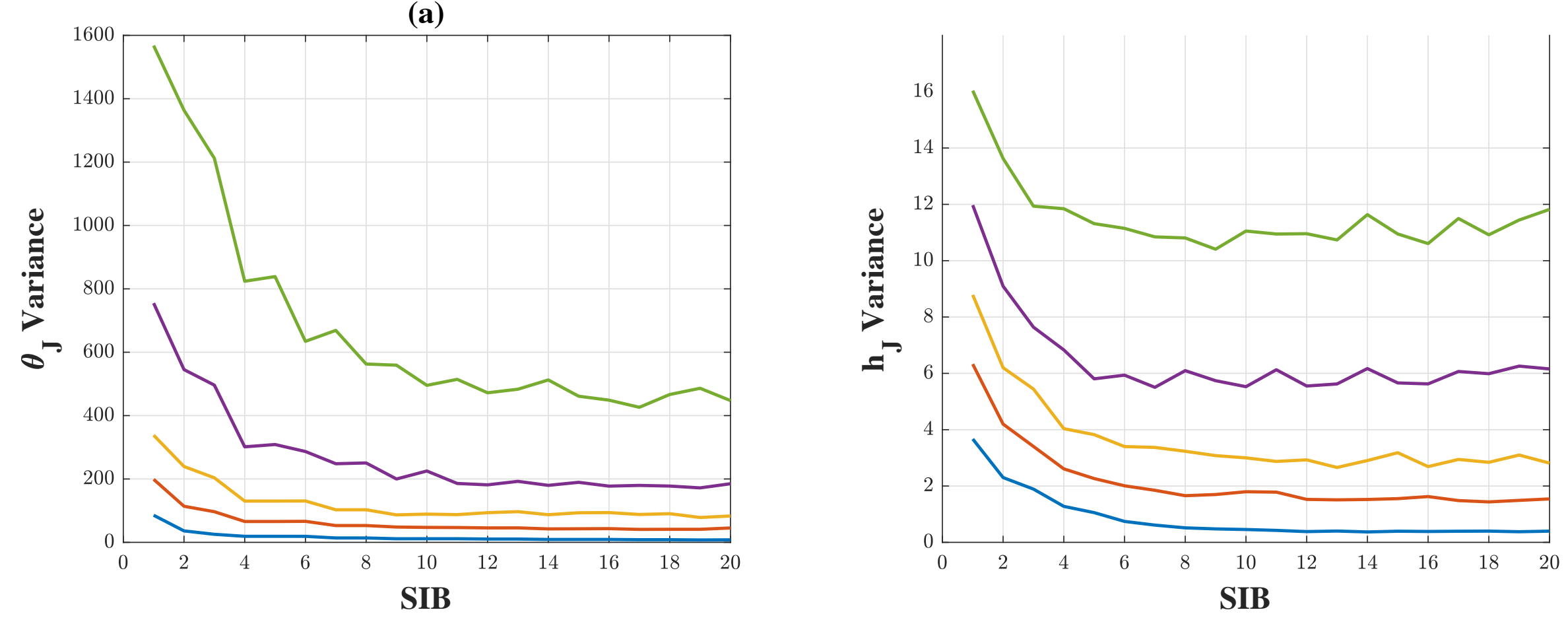

(c)


$-\sigma_{\mathrm{AB}} / \mathrm{d}_{\mathrm{AB}}=0.005-\sigma_{\mathrm{AB}} / \mathrm{d}_{\mathrm{AB}}=0.025$

$-\sigma_{\mathrm{AB}} / \mathrm{d}_{\mathrm{AB}}=0.05$

$\sigma_{\mathrm{AB}} / \mathrm{d}_{\mathrm{AB}}=0.1-\sigma_{\mathrm{AB}} / \mathrm{d}_{\mathrm{AB}}=0.25$ 